\title{
Inhibition of PINK1/Parkin-dependent mitophagy sensitizes multidrug-resistant cancer cells to B5G1, a new betulinic acid analog
}

\author{
Nan Yao ${ }^{1,2}$, Chenran Wang ${ }^{1,2}$, Nan Hu ${ }^{1,2}$, Yingjie Li ${ }^{1,2}$, Mingqun Liu ${ }^{1,2}$, Yuhe Lei ${ }^{1,2}$, Minfeng Chen ${ }^{1,2}$, Liping Chen ${ }^{1,2}$, \\ Chen Chen', Ping Lan', Weimin Chen $\mathbb{1}^{1}$, Zhesheng Chen ${ }^{1,3}$, Dengrui Fu', Wencai Ye ${ }^{1,2}$ and Dongmei Zhang ${ }^{1,2}$
}

\begin{abstract}
Betulinic acid (BA) and its derivatives are a class of high-profile drug candidates, but their anticancer effects on resistant cancer have rarely been reported. Although a few studies indicated mitophagy is related with drug resistance, its role in different cancer types and anticancer agents treatment remains largely unclear. Here, we find that B5G1, a new derivative of BA, induces cell death in multidrug resistant cancer cells HepG2/ADM and MCF-7/ADR through mitochondrial-apoptosis pathway. B5G1 also triggers mitophagy independent on Atg5/Beclin 1. Further mechanistic study indicates that B5G1 upregulates PTEN-induced putative kinase 1 (PINK1) to recruit Parkin to mitochondria followed by ubiquitination of Mfn2 to initiate mitophagy. Inhibition of mitophagy by PINK1 siRNA, mdivi-1, or bafilomycin A1 (Baf A1) promotes B5G1-induced cell death. In addition, ROS production and mitochondrial damage in B5G1-treated HepG2/ADM cells cause mitochondrial apoptosis and mitophagy. In vivo study shown that B5G1 dramatically inhibits HepG2/ADM xenograft growth accompanied by apoptosis and mitophagy induction. Together, our results provide the first demonstration that B5G1, as a novel mitophagy inducer, has the potential to be developed into a drug candidate for treating multidrug resistant cancer.
\end{abstract}

\section{Introduction}

Multidrug resistance (MDR) mediated by ATP-binding cassette $(\mathrm{ABC})$ transporters is the primary obstacle to successful cancer chemotherapy ${ }^{1}$. Although numerous MDR reversal agents targeting $\mathrm{ABC}$ transporters have been developed, poor efficacy and severe side effects have caused their failure in clinical trials ${ }^{2,3}$. Therefore, the need to explore novel chemotherapeutic agents and effective strategies against resistant cancers is urgent.

\footnotetext{
Correspondence: Dongmei Zhang (dmzhang701@jnu.edu.cn)

${ }^{1}$ College of Pharmacy, Jinan University, 510632 Guangzhou, China

${ }^{2}$ Guangdong Province Key Laboratory of Pharmacodynamic Constituents of

Traditional Chinese Medicine and New Drugs Research, Jinan University,

510632 Guangzhou, China

Full list of author information is available at the end of the article.

Edited by G.M. Fimia

These authors contributed equally: Nan Yao, Chenran Wang, and Nan Hu
}

Mitophagy is a type of selective autophagy that promotes mitochondrial turnover and prevents the accumulation of dysfunctional mitochondria to maintain cellular homeostasis. Recently, several reports suggested that mitophagy contribute to chemotherapeutic efficacy or drug resistance in cancer. In melanoma cells, inhibition of the mitochondrial respiratory chain by BAY $87-2243$ induced mitophagy-dependent necroptosis and ferropto$\mathrm{sis}^{4}$. Targeting orphan nuclear receptor TR3 with a small molecule led to permeability transition pore opening, which results in excessive mitophagy and irreversible A375 cell death ${ }^{5}$. Selenite induced superoxide anionmediated mitophagic cell death in glioma cells ${ }^{6}$. On the other hand, Doxorubicin (Dox)-induced mitophagy contributes to drug resistance in HCT8 human colorectal cancer stem cells. Inhibiting mitophagy by silencing 
BNIP3L enhanced Dox sensitivity in colorectal cancer stem cells ${ }^{7}$. Liensinine sensitized breast cancer cells to chemotherapy by mitophagy inhibition through DNM1Lmediated mitochondrial fission ${ }^{8}$. Although mitophagy is related with drug resistance, its role in different cancer types and anticancer agents treatment remains largely unclear.

Currently, a mechanism of mitophagy based on PTENinduced putative kinase 1 (PINK1) and Parkin, an E3 ubiquitin ligase, is widely accepted. When mitochondrial membrane potential (MMP) is impaired by ROS, irradiation, or chemotherapeutic agents, PINK1 is stabilized on the outer mitochondrial membrane, leading to Parkin recruitment to damaged mitochondria ${ }^{9}$. Mitochondrialanchored Parkin is phosphorylated at Ser65 by PINK1 and performs ubiquitination; this process results in further ubiquitination of other mitochondrial proteins, such as VDAC, TOM20, and Mfn2, to facilitate impaired mitochondria recognition ${ }^{10}$. However, Parkin-independent mitophagy has also been reported ${ }^{11,12}$. As a selective type of autophagy, the formation of mitochondrial autophagosomes is also subject to the regulatory mechanisms of autophagy. This process depends on autophagy-related proteins, such as Beclin 1, Atg5, and Atg12, for the formation, elongation, and closure of LC3-coated phagophores $^{13}$. However, the roles of autophagy regulatory proteins differ in various types of cancers, and their underlying mechanisms are complicated and not fully understood. Therefore, the discovery of small molecule probes modulating mitophagy will be highly significant for revealing the molecular mechanisms of mitophagy.

Natural products and their derivatives are primary sources of anticancer agents that act via novel mechanisms. Betulinic acid (BA) and its derivatives, a class of high-profile bioactive agents, exhibit broad-spectrum anticancer activities, but little attention has been paid to their effects on multidrug-resistant cancer ${ }^{14-17}$. Accumulating evidence demonstrates that the mechanisms underlying cell death induced by BA and its derivatives are complicated and dependent on the cancer cell type. These compounds induce apoptosis in multiple myeloma, prostate cancer, and cervical cancer cells via multiple signaling pathways, such as the STAT3, NF- $\mathrm{kB}$, and PI3K/ Akt pathways ${ }^{18-20}$. Recent several studies have shown that BA and B10, a glycosylated derivative of BA, induce cell death by inhibiting autophagic flux in microglia, glioblastoma, and multiple myeloma cells ${ }^{21-23}$. In contrast, a few studies have reported that BA-induced autophagy as a pro-survival mechanism in colorectal, cervical, and breast cancer cells ${ }^{24,25}$. This pro-survival mechanism has been associated with p53 or the opening of the mitochondrial permeability transition pore ${ }^{24}$. However, the role of mitophagy has still not been investigated in cancer cells treated with BA or its derivatives.
In this study, we found that a new derivative of BA, B5G1, had potent anticancer activity towards multidrugresistant cancer cells HepG2/ADM and MCF-7/ADR. B5G1 induced ROS production and mitochondrial dysfunction, thereby triggering mitophagy in a manner dependent on PINK1 and Parkin but not Atg5 and Beclin 1 , and mitophagy inhibition promotes B5G1-induced apoptosis in drug-resistant cancer cells.

\section{Results}

B5G1 inhibits the proliferation of multidrug-resistant cancer cells via induction of mitochondrial apoptosis

B5G1 cytotoxicity against HepG2, HepG2/ADM, MCF7, and MCF-7/ADR cells was evaluated by MTT assay and LDH assay. B5G1 showed selective cytotoxicity towards multidrug-resistant cancer cells HepG2/ADM and MCF7/ADR but not their parent cells HepG2 and MCF-7 (Fig. 1a, b; Supplementary Fig. S1B and C). B5G1 decreased HepG2/ADM cell survival in a concentrationand time-dependent manner (Fig. 1a, c). It also inhibited the proliferation of HepG2/ADM colonies (Fig. 1d). HepG2/ADM is a multidrug-resistant cell line overexpressing ABCB1 (Supplementary Fig. S1D). ABCB1substrates, such as Dox and vincristine (VCR), showed little cytotoxicity against HepG2/ADM cells. Determining whether B5G1 is a substrate of drug-resistant cells is important. As shown in Fig. 1e, VRP, a specific inhibitor of $A B C B 1$, had no effect on B5G1-induced cell death, indicating that B5G1 is not a substrate of ABCB1.

Apoptosis induction is frequently reported in some cancer cell lines treated with BA or its derivatives. Similarly, the apoptotic rates increased in a time-dependent manner upon B5G1 treatment in HepG2/ADM (Fig. 1f) and MCF-7/ADR cells (Supplementary Fig. S1E). Caspase-9, caspase-3, and PARP cleavage was increased significantly after B5G1 treatment, but there was no significant change in caspase-8, a key mediator of the death receptor apoptosis pathway in HepG2/ADM (Fig. 1g) and MCF-7/ADR cells (Supplementary Fig. S1F). Moreover, cyto $c$ was released from the mitochondria into the cytoplasm after B5G1 treatment (Fig. 1h; Supplementary Fig. S1G). Taken together, these results indicate that B5G1 induces multidrug-resistant cancer cell death via a mitochondrial apoptotic pathway.

\section{B5G1 induces mitophagy in multidrug-resistant cancer cells}

As BA-induced autophagy in several cancer lines, we speculated that B5G1 would also induce autophagy in drug-resistant cancer cells. As expected, after B5G1 treatment for $12 \mathrm{~h}, \mathrm{MDC}$-positive autophagic vesicles were present in HepG2/ADM cells (Fig. 2a), and LC3 accumulation was found (Fig. 2a, b). Moreover, upon B5G1 treatment, bilayer membrane-bound autophagosomes 
A
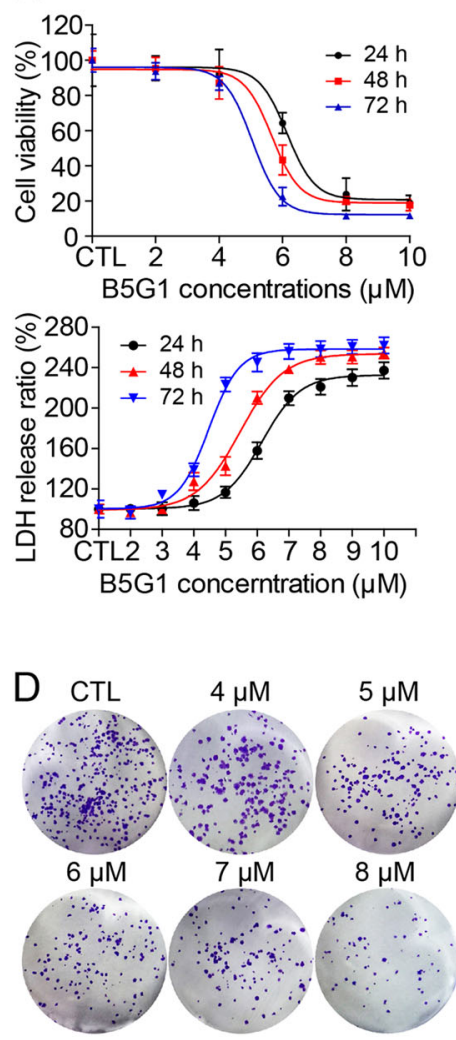

$\mathrm{F}$

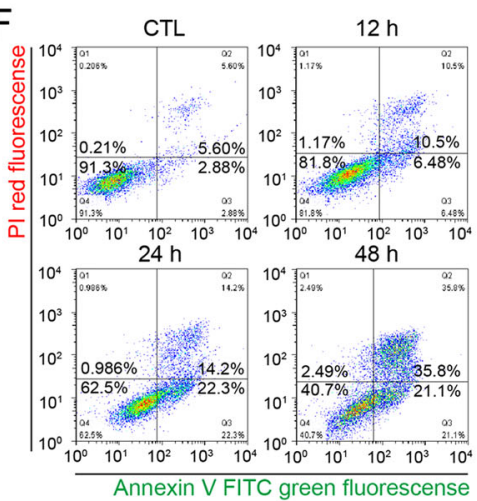

$\mathrm{H}$

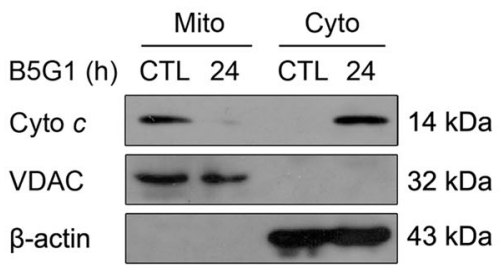

B

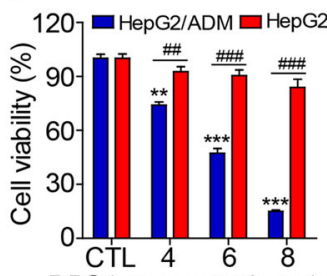

B5G1 concentrations $(\mu \mathrm{M})$

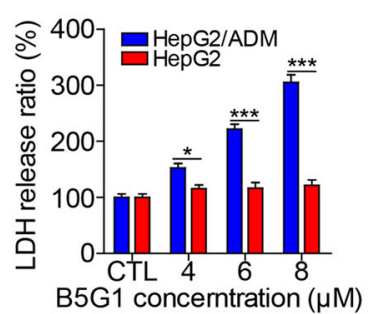

C
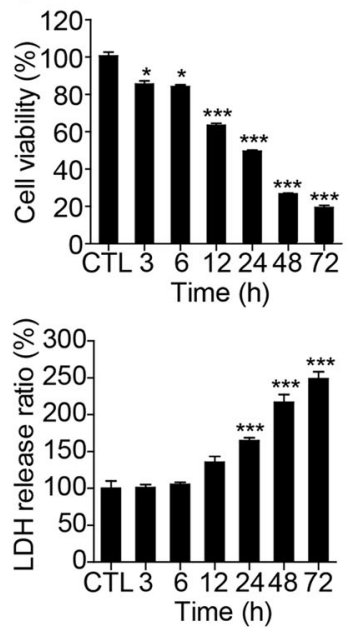

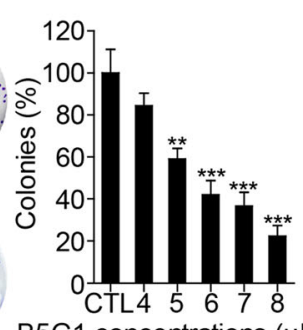

$\mathrm{E}$

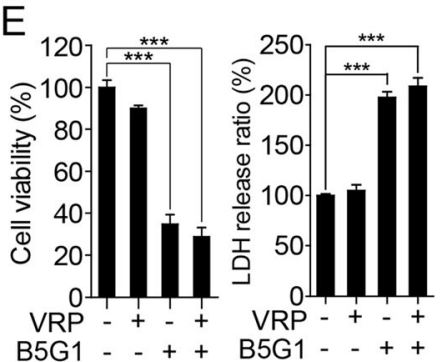

B5G1 concentrations $(\mu \mathrm{M})$

$\mathrm{B} 5 \mathrm{G} 1 \ldots++\mathrm{B} 5 \mathrm{G} 1 \ldots++$

G

\begin{tabular}{|c|c|c|}
\hline B5G1 (h) & $\begin{array}{lllll}\text { CTL } & 6 & 12 & 24 & 48\end{array}$ & \\
\hline Caspase-9 & $00--1$ & $47 \mathrm{kDa}$ \\
\hline Cleaved caspase- 9 & $-\pi-0$ & $\begin{array}{l}37 \mathrm{kDa} \\
35 \mathrm{kDa}\end{array}$ \\
\hline Caspase-8 & 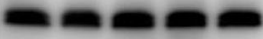 & $57 \mathrm{kDa}$ \\
\hline Cleaved caspase-8 & & $43 \mathrm{kDa}$ \\
\hline Cleaved caspase-8 & & $18 \mathrm{kDa}$ \\
\hline Caspase-3 & $=2==$ & $35 \mathrm{kDa}$ \\
\hline Cleaved caspase-3 & $-\cdots$ & $17 \mathrm{kDa}$ \\
\hline PARP & $00--1$ & $116 \mathrm{kDa}$ \\
\hline Cleaved PARP & $--\infty$ & $89 \mathrm{kDa}$ \\
\hline$\beta$-actin & $-\infty-0$ & $43 \mathrm{kDa}$ \\
\hline
\end{tabular}

Fig. 1 (See legend on next page.)

were observed by transmission electron microscopy, further indicating that the induction of autophagy is mediated by B5G1 (Fig. 2c).

Both MitoTracker red and Mitotracker green staining showed that the number of mitochondria decreased gradually (Fig. 2d), and the mitochondrial proteins COX IV, TOM20, and Mfn2 were degraded in a timedependent manner in HepG2/ADM cells (Fig. 2e), as well as MCF-7/ADR cells which were inhibited by Baf A1 (Supplementary Fig. S2A and B). These data indicated 
(see figure on previous page)

Fig. 1 B5G1 inhibits the proliferation of HepG2/ADM cells via induction of mitochondrial apoptosis. a HepG2/ADM cells were treated with different concentrations of B5G1 for 24, 48, and $72 \mathrm{~h}$. Cell viability was determined by MTT and LDH assay. $\mathbf{b}$ HepG2/ADM and HepG2 cells were treated with the indicated concentrations of B5G1 for $48 \mathrm{~h}$, and cell viability was determined by MTT and LDH assay $(n=3) .{ }^{* *} P<0.01,{ }^{* * * *} P<0.001$ vs CTL (HepG2/ADM), ${ }^{\# \# P} P<0.01,{ }^{\# \# \# P} P<0.001$ (MTT assay); ${ }^{*} P<0.05,{ }^{* * *} P<0.001$ (LDH assay). c HepG2/ADM cells were treated with B5G1 (6 $\mu$ M) for the indicated times, and cell viability was determined by MTT and LDH assay $(n=3) .{ }^{*} P<0.05,{ }^{* * *} P<0.001$ vs CTL. d HepG2/ADM cells were treated with the indicated concentrations of B5G1 for $24 \mathrm{~h}$. Colonies were visualized by crystal violet staining and counted manually $(n=3)$. Magnification: $\times 200$; ${ }^{* *} P<0.01,{ }^{* * *} P<0.001$ vs CTL. e HepG2/ADM cells were treated with B5G1 $(6 \mu \mathrm{M})$ in the presence or absence of VRP $(50 \mu \mathrm{M})$ for $48 \mathrm{~h}$, cell viability was measured by MTT and LDH assay $(n=3) .{ }^{* * *} P<0.001$. $\mathbf{f}$ The apoptosis rates of HepG2/ADM cells treated with B5G1 $(6 \mu$ M) were detected by flow cytometry. $\mathbf{g}$ Apoptosis-related proteins expression level of HepG2/ADM cells treated with B5G1 $(6 \mu \mathrm{M})$ for the indicated times were analyzed by western blotting. $\beta$-actin was used as a loading control. $\mathbf{h}$ Cell lysates of HepG2/ADM cells treated with B5G1 ( $6 \mu \mathrm{M})$ for $24 \mathrm{~h}$ were divided into cytoplasmic fractions and mitochondrial fractions. Cyto $\mathrm{c}$ translocation was measured by western blotting. $\beta$-actin and VDAC were used as loading controls for cytoplasm and mitochondria, respectively

that mitochondrial proteins are likely to be eliminated by mitophagy rather than inhibition of de nove protein synthesis. To further confirm the occurrence of mitophagy, we transfected HepG2/ADM cells with mitoKeima plasmids. Mito-Keima is a pH-sensitive fluorescent protein located in mitochondria that can be used to identify mitochondrial movement from the cytoplasm (green) to lysosomes (red). As shown in Fig. 2f, red spots appeared in the cytoplasm after B5G1 treatment, indicating that mitochondria tended to form autolysosomes. In addition, the colocalization of mitochondria with LC3 or LAMP1 (a lysosome marker) further demonstrated mitophagy induction by B5G1 in HepG2/ADM and MCF7/ADR cells (Fig. 2g, h; Supplementary Fig. S2C and D).

\section{B5G1-induced mitophagy is regulated by a nonclassical autophagy pathway}

Because Beclin 1 and Atg5 are two well-known molecules involved in autophagy regulation ${ }^{27}$, we determined the influence of B5G1 on these two regulators. Notably, the expression levels of these two proteins showed no apparent change upon B5G1 treatment (Fig. 3a). Knocking down the expression of Atg 5 or Beclin 1 had no influence on LC3-II levels (Fig. 3b). Furthermore, B5G1-induced colocalization of clustered mitochondria with LC3 or lysosomes was not affected by Beclin 1 siRNA pretreatment (Fig. 3c, d). Futhermore, 3-MA, a Class III PI3K inhibitor, also failed to abolish the colocalization of mitochondria with LC3 (Fig. 3e). These results implied that the mitophagy caused by B5G1 is regulated by a nonclassical pathway that is independent of Beclin 1 and Atg5.

\section{B5G1-induced mitophagy is mediated by PINK1/Parkin}

PINK1 and Parkin are two key factors that regulate mitophagy. Parkin is translocated to the mitochondrial surface through the upregulation of PINK1, which in turn initiates mitophagy ${ }^{28}$. We found that PINK1 was significantly upregulated after B5G1 treatment in HepG2/ ADM (Fig. 4a) and MCF-7/ADR cells (Supplementary Fig. S2E). Parkin was translocated from the cytoplasm to the mitochondria, and p-Parkin (Ser65) was upregulated by B5G1 in HepG2/ADM (Fig. 4a, b) and MCF-7/ADR cells (Supplementary Fig. S2F). A coimmunoprecipitation assay was performed to further investigate the interaction between PINK1 and Parkin. As shown in Fig. 4c, levels of PINK1 and the Parkin complex increased upon B5G1 treatment for $12 \mathrm{~h}$ in HepG2/ADM cells. These results indicated that B5G1-mediated mitophagy is associated with PINK1 and Parkin. To further confirm the role of PINK1 and Parkin in B5G1-induced mitophagy, siRNAmediated PINK1 knockdown was conducted. In contrast to control siRNA, PINK1 siRNA significantly abolished mitochondrial colocalization with LC3 in HepG2/ADM cells (Fig. 4d, e). Knockdown of Parkin also get the same effect (Fig. 4f, g). Taken together, these findings indicate that B5G1 induces mitochondrial PINK1 upregulation to recruit Parkin, thereby initiating mitophagy.

\section{B5G1 induces Mfn2 ubiquitylation and mitochondrial anchoring of the autophagy adaptor p62/SQSTM1}

Ubiquitylation of mitochondrial proteins, such as VDAC, Mfn2, and TOM20, is required for the recognition of damaged mitochondria by autophagy-related proteins ${ }^{10}$. In this process, Parkin is ubiquitylated by PINK1 first, so we detected Parkin ubiquitylation by western blotting in HepG2/ADM cells. Upon B5G1 treatment, ubiquitin modification of Parkin was detected by CO-IP assays (Fig. 5a). Moreover, ubiquitin modification of Mfn2 (Fig. 5b), but not TOM20 and VDAC (data not shown), was detected after B5G1 treatment. The ubiquitin-binding protein $\mathrm{p} 62 / \mathrm{SQSTM} 1$ targets ubiquitylated mitochondrial proteins and binds them to form the autophagosomes that act in mitophagy. To investigate whether p62 is associated with B5G1-induced mitophagy, we analyzed the cellular localization of p62. As shown in Fig. 5c, p62 showed significant colocalization with mitochondria after B5G1 treatment for $12 \mathrm{~h}$, and p62 siRNA pretreatment abolished the colocalization of mitochondria and LC3 in HepG2/ADM cells (Fig. 5d, e). In summary, these data show that Mfn2 ubiquitylation and p62 recruitment to mitochondria take part in PINK1/Parkin-mediated mitophagy in B5G1-treated resistant cancer cells. 

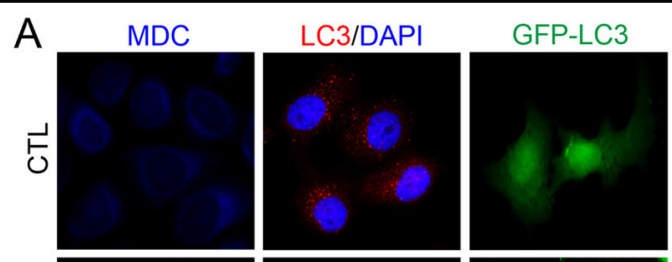

B
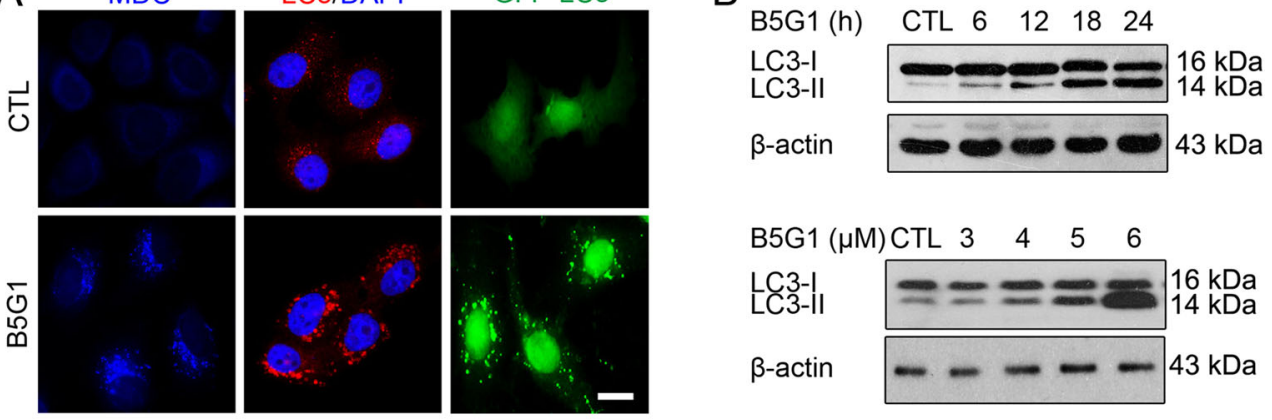

C

CTL

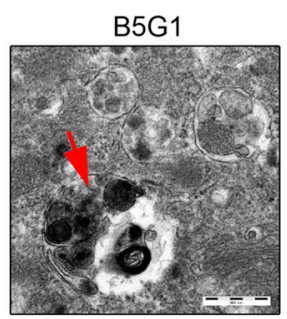

D
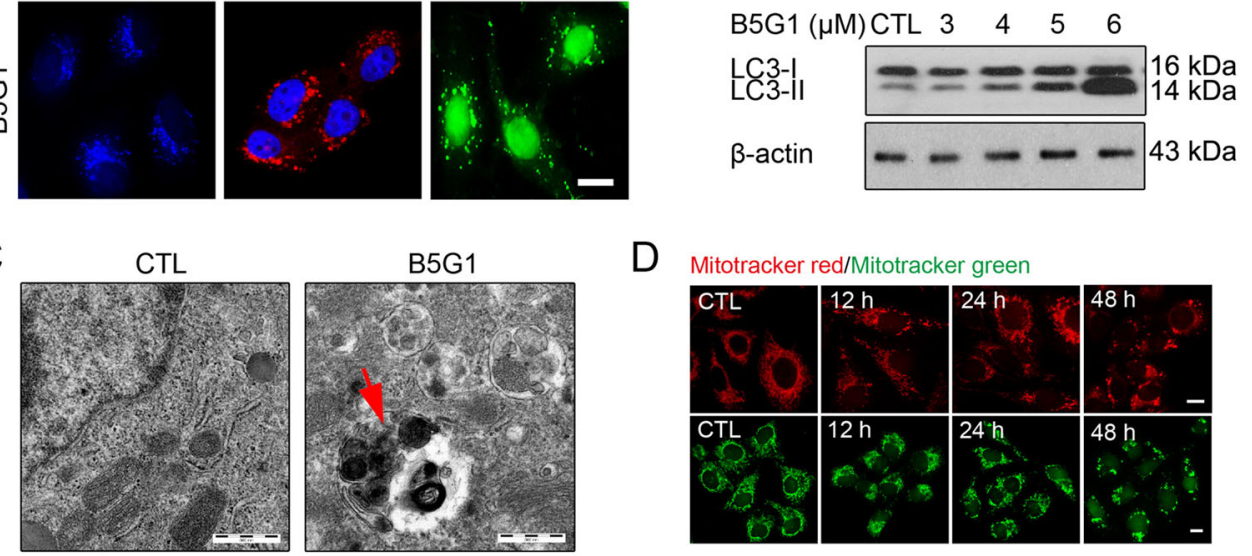

Mitotracker red/Mitotracker green
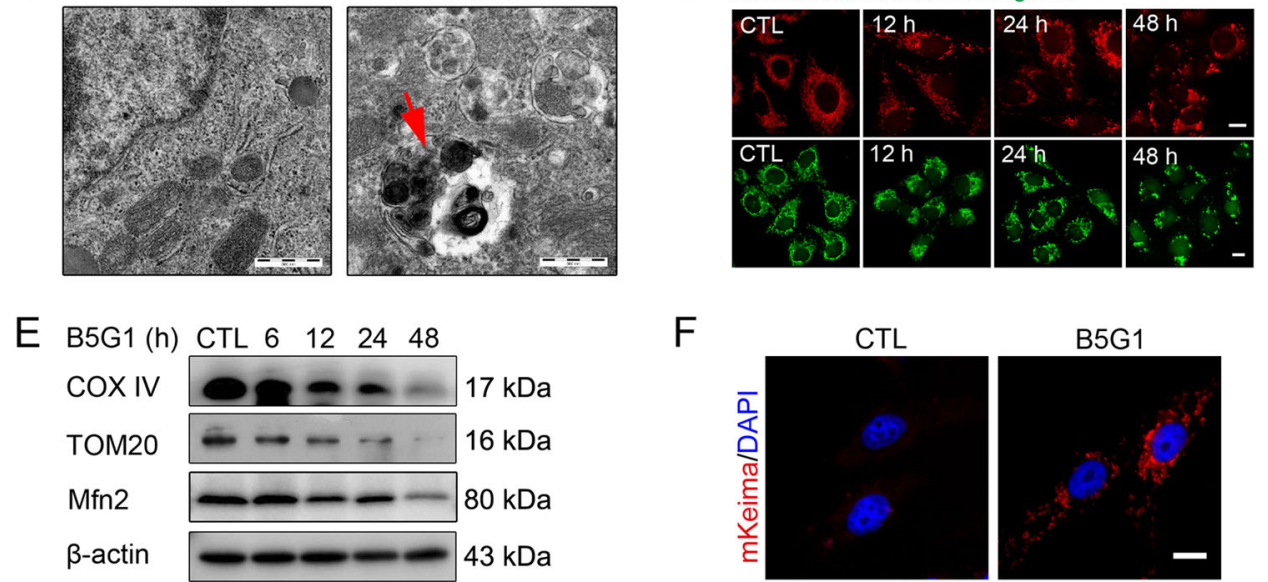

F
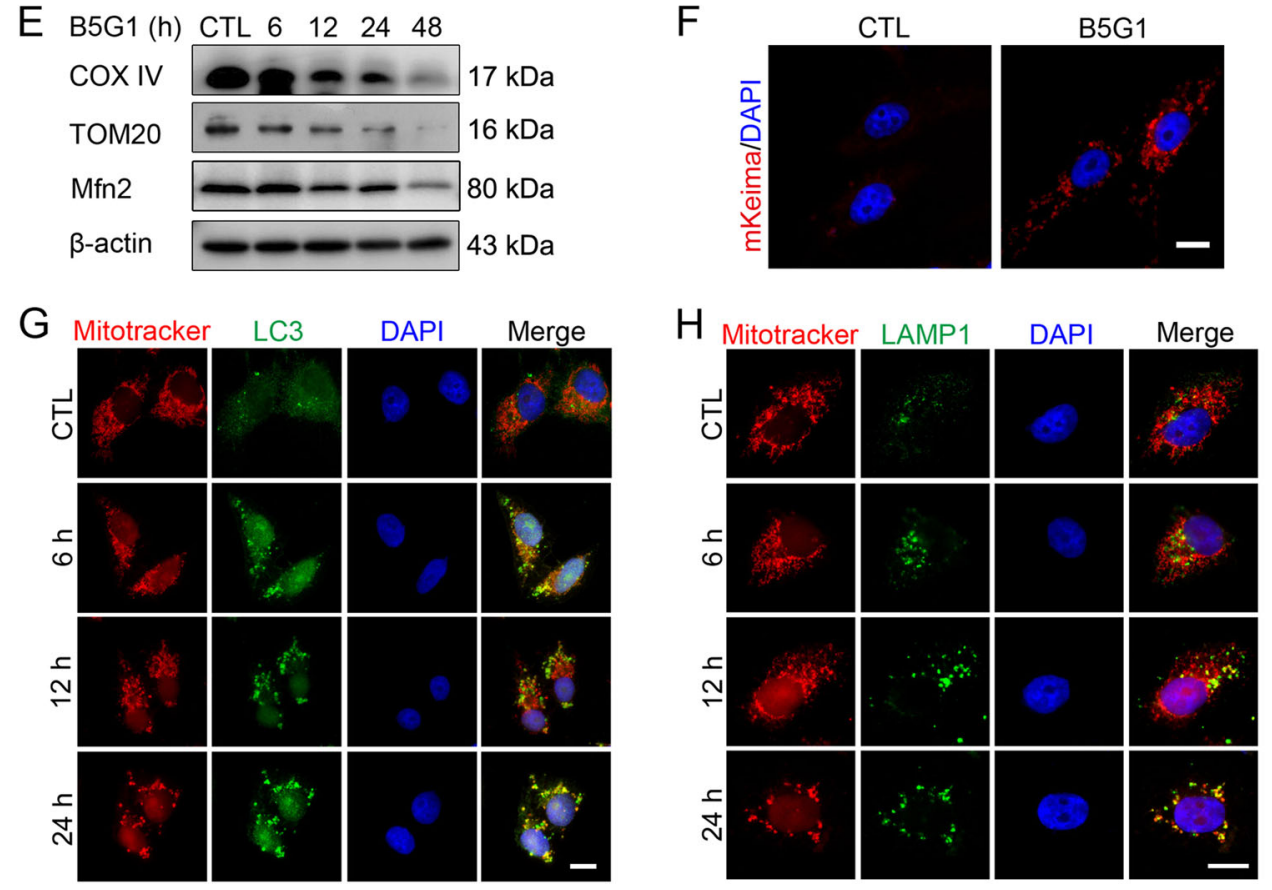

Fig. 2 B5G1 induces mitophagy in HepG2/ADM cells. a After B5G1 treatment ( $6 \mu \mathrm{M})$ for $24 \mathrm{~h}$, HepG2/ADM cells were stained with MDC (50 $\mu M)$ or immunostained with a LC3 antibody. For the GFP-LC3 assay, HepG2/ADM cells were transfected with the GFP-LC3 plasmid for $24 \mathrm{~h}$, followed by treatment with B5G1 $(6 \mu \mathrm{M})$ for $24 \mathrm{~h}$. The fluorescence was observed by a fluorescence microscope. Magnification: $\times 630$, scale bar: $10 \mu \mathrm{m}$. b HepG2/ ADM cells were treated with B5G1 as indicated, and LC3 expression level was then detected by western blotting. $\beta$-actin was used as a loading control. c After treatment with B5G1 $(6 \mu \mathrm{M})$ for $24 \mathrm{~h}$, the ultrastructure of HepG2/ADM cells was observed by a transmission electron microscopy. Magnification: ×8900; Scale bar: $500 \mathrm{~nm}$. d HepG2/ADM cells were treated with B5G1 (6 $\mu$ M) for 0, 12, 24, or $48 \mathrm{~h}$ and then stained with MitoTracker Red $(200 \mathrm{nM})$ or MitoTracker Green (200 nM). The fluorescence was observed by a fluorescence microscope. Magnification: $\times 630 ;$ scale bar: $10 \mu \mathrm{m}$. e HepG2/ADM cells were exposed to B5G1 $(6 \mu \mathrm{M})$ for the indicated times. Mitochondrial proteins expression levels were measured by western blotting. $\beta$-actin was used as a loading control. $\mathbf{f}$ HepG2/ADM cells were transfected with the mKeima-Red-Mito-7 plasmid, followed by treatment with B5G1 $(6 \mu \mathrm{M})$ for $24 \mathrm{~h}$. The Fluorescence was detected by a fluorescence microscope. Magnification: $\times 630 ;$ scale bar: $10 \mu \mathrm{m}$. $\mathbf{g}, \mathbf{h}$ After treatment with B5G1 $(6 \mu \mathrm{M})$ for the indicated times, HepG2/ADM cells were stained with MitoTracker red (200 nM) and immunostained with a LC3 or LAMP1 antibody. Mitochondrial colocalization with LC3 or LAMP1 was observed by a fluorescence microscope. Magnification: $\times 630$; scale bar: $10 \mu \mathrm{m}$ 


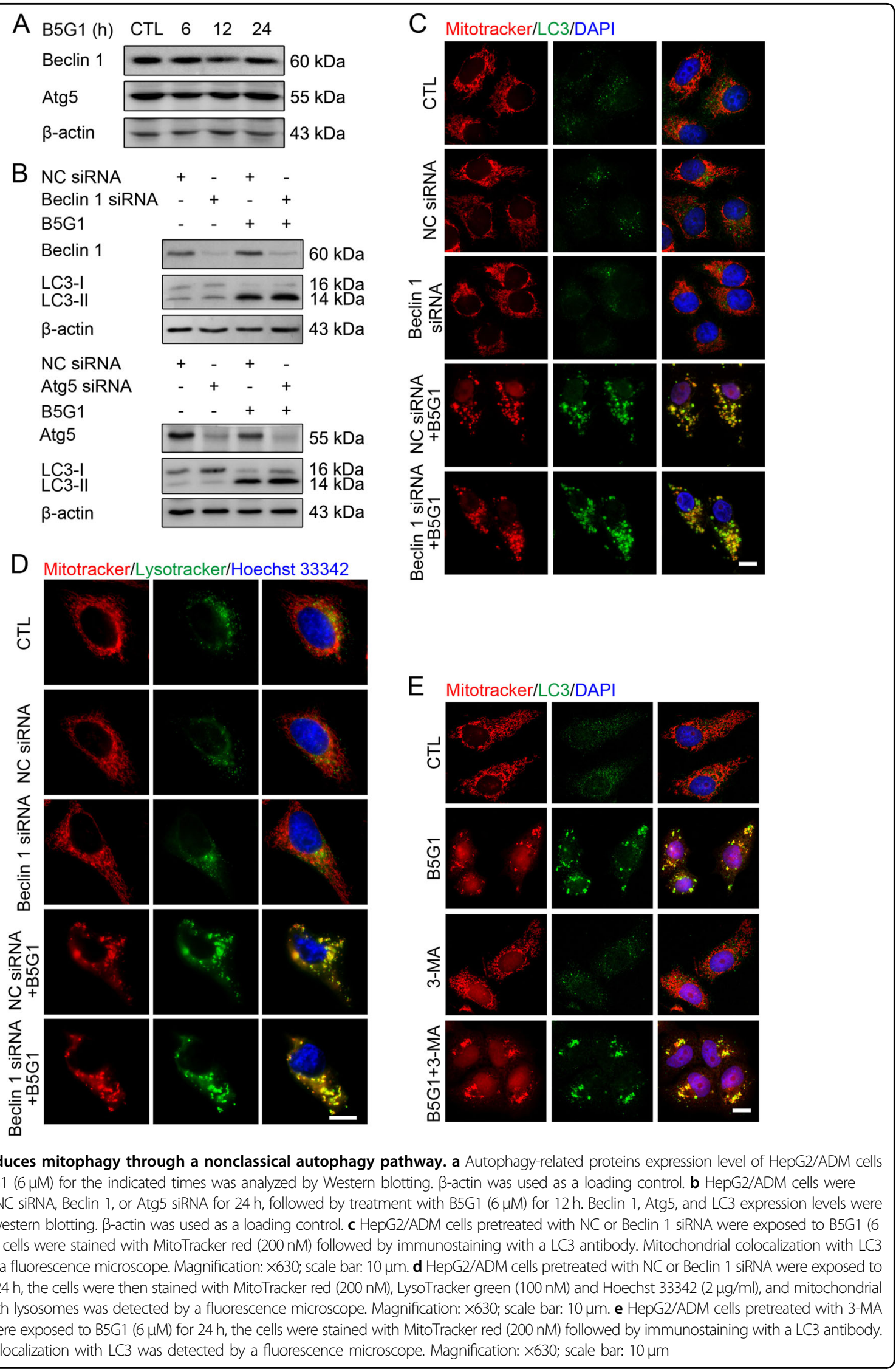




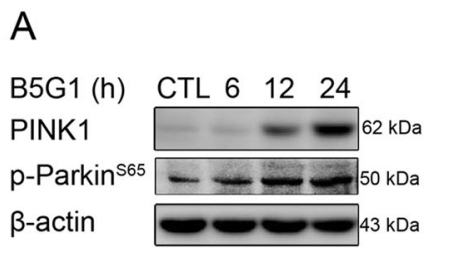

C

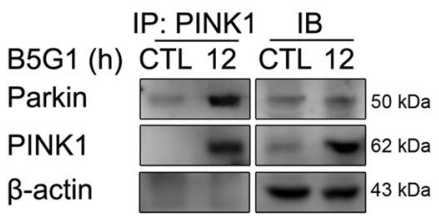

E
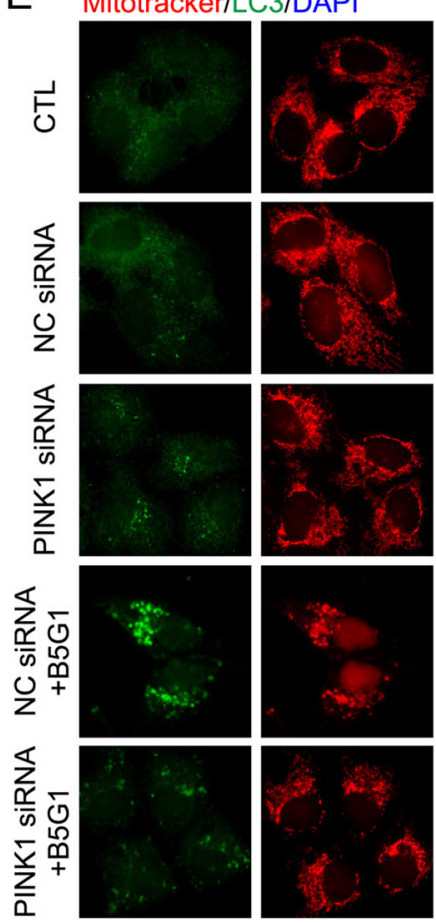

\section{B}

B5G1 (h)

Parkin

$\beta$-actin

VDAC

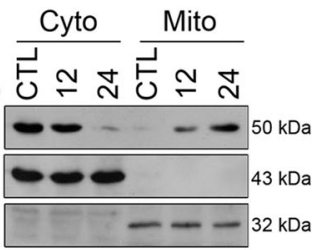

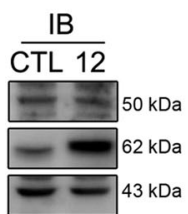

D
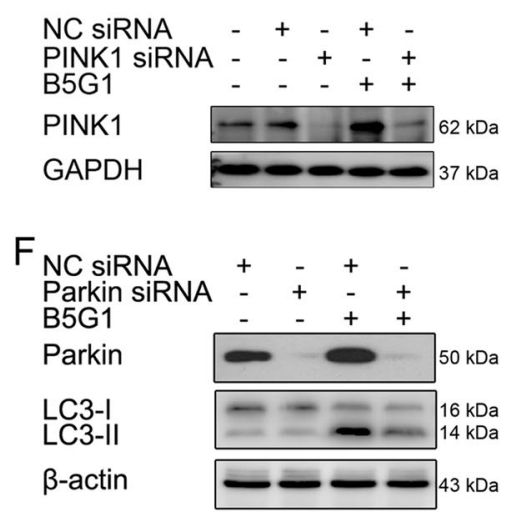

Fig. 4 B5G1-induced mitophagy is mediated by the PINK1/Parkin pathway. a After treatment with B5G1 (6 $\mu$ M) for the indicated times, PINK1 and p-Parkin (Ser65) expression levels were detected by western blotting. $\beta$-actin was used as a loading control. b HepG2/ADM cells treated with B5G1 $(6 \mu \mathrm{M})$ for the indicated times were lysed and then separated into mitochondrial fractions and cytosolic fractions. Parkin translocation was measured by Western blotting. $\beta$-actin and VDAC were used as loading controls for the cytosolic and mitochondrial proteins, respectively. c After treatment with B5G1 $(6 \mu \mathrm{M})$ for $12 \mathrm{~h}$, HepG2/ADM cells were lysed with CO-IP lysis buffer, and the interaction between PINK1 and Parkin was measured by Co-IP assay. The asterisk indicated the band of Parkin. $\mathbf{d}$ HepG2/ADM cells were pretreated with NC or PINK1 siRNA, followed by treatment with B5G1 $(6 \mu \mathrm{M})$ for $12 \mathrm{~h}$. PINK1 expression levels were detected by western blotting. GAPDH was used as a loading control. e HepG2/ ADM cells were transfected with NC or PINK1 siRNA for $24 \mathrm{~h}$ and then treated with B5G1 $(6 \mu \mathrm{M})$ for another $24 \mathrm{~h}$. Mitochondrial colocalization with LC3 was observed by a fluorescence microscope. Magnification: ×630; scale bar: $10 \mu \mathrm{m}$. $\mathbf{f}$ HepG2/ADM cells were transfected with NC or Parkin siRNA for $24 \mathrm{~h}$ and then treated with B5G1 $(6 \mu \mathrm{M})$ for $12 \mathrm{~h}$. Parkin and LC3 expression levels were detected by western blotting. $\beta$-actin was used as a loading control. $\mathbf{g ~ H e p G 2 / A D M}$ cells were transfected with NC or Parkin siRNA for $24 \mathrm{~h}$ and then treated with B5G1 $(6 \mu \mathrm{M})$ for $24 \mathrm{~h}$. Mitochondrial colocalization with LC3 was detected by immunofluorescence. Magnification: ×630; scale bar: $10 \mu \mathrm{m}$

\section{Mitophagy inhibition counterbalances apoptosis and sensitizes multidrug-resistant cancer cells to B5G1}

As mitophagy acts as "a double-edged sword" in cancer, we next investigated the interaction of B5G1-induced mitophagy and apoptosis in drug-resistant cancer cells using PINK1 siRNA, a mitophagy inhibitor, mdivi-1, and a lysosome inhibitor, Baf A1. PINK1 siRNA pretreatment increased B5G1-induced cell death as well as caspase-3, 


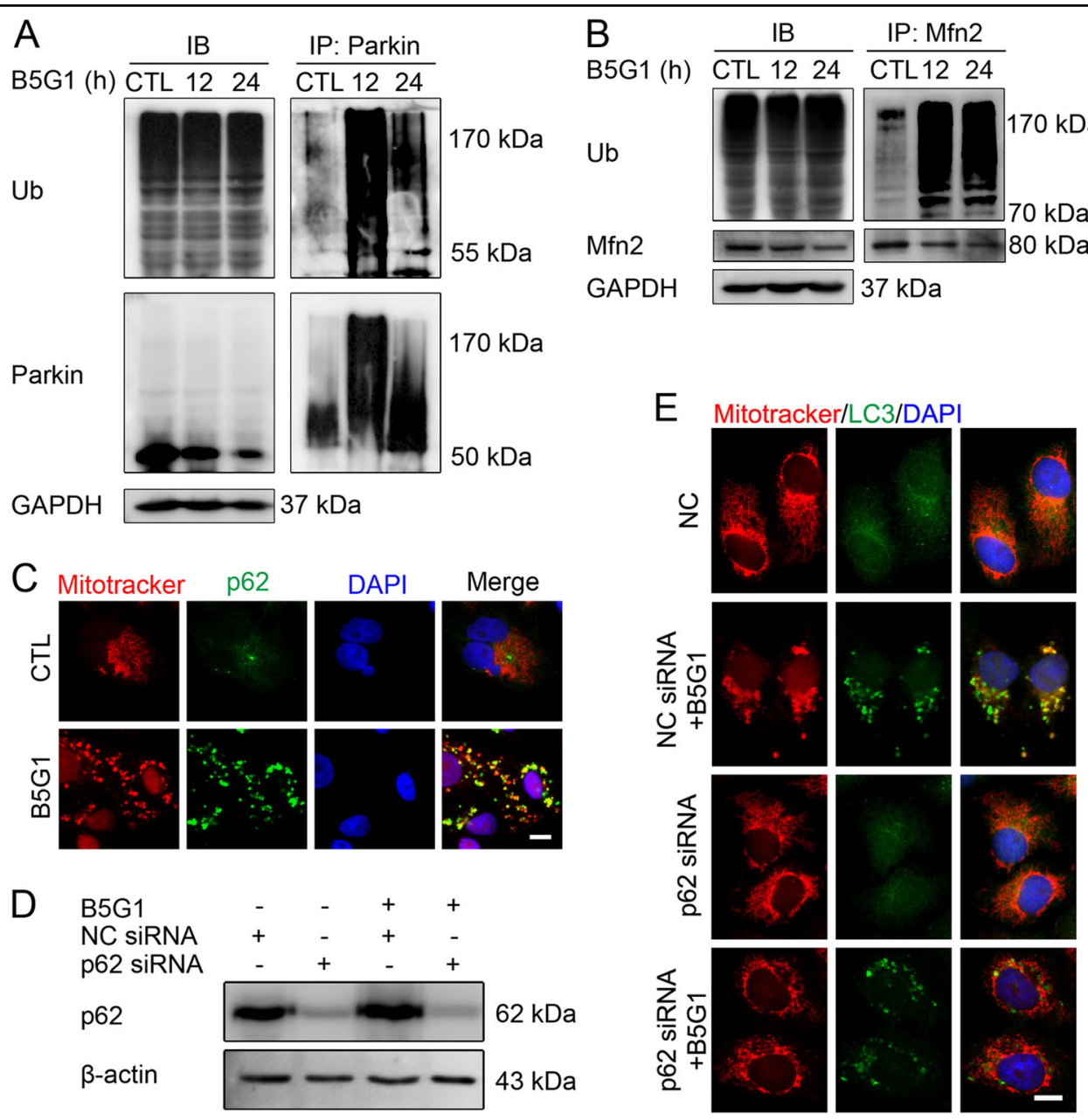

Fig. 5 B5G1 induces Mfn2 ubiquitination and mitochondrial anchoring of the autophagy adaptor p62/SQSTM1. a, b HepG2/ADM cells were treated with B5G1 $(6 \mu \mathrm{M})$ for the indicated times, Parkin and Mfn2 ubiquitination were detected by coimmunoprecipitation. c HepG2/ADM cells were treated with B5G1 $(6 \mu \mathrm{M})$ for $24 \mathrm{~h}$, and mitochondrial colocalization with p62 was detected by immunofluorescence. Magnification: $\times 630 ;$ scale bar: $10 \mu \mathrm{m}$. $\mathbf{d}$ HepG2/ADM cells were transfected with NC or p62 siRNA before treatment with B5G1 (6 $\mu \mathrm{M})$ for $12 \mathrm{~h}$. p62 expression level was detected by western blotting. $\beta$-actin was used as a loading control. e HepG2/ADM cells were transfected with NC or p62 siRNA and then treated with B5G1 $(6 \mu \mathrm{M})$ for $24 \mathrm{~h}$. Mitochondrial colocalization with LC3 was detected by immunofluorescence. Magnification: ×630; scale bar: $10 \mu \mathrm{m}$

caspase-9, and PARP cleavage in HepG2/ADM cells (Fig. 6a, b). Mdivi-1, a mitophagy inhibitor ${ }^{4,29}$, was used to further investigate the role of B5G1-induced mitophagy. Pretreatment with mdivi-1 inhibited the B5G1-induced colocalization of mitochondria with LC3 (Fig. 6c). The corresponding apoptotic proteins were further activated in the presence of mdivi-1 in B5G1-treated HepG2/ADM cells (Fig. 6d). Furthermore, mdivi-1 pretreatment increased B5G1-induced apoptosis and cell death in HepG2/ADM cells (Fig. 6d, e). Baf A1, which inhibits the fusion of autophagosomes with lysosomes, also sensitized HepG2/ADM cells to B5G1 (Fig. 6f). In addition, PINK1 depletion and Baf A1 pretreatment also sensitized MCF7/ADR cells to B5G1 treatment (Supplementary Fig. S3AC). In conclusion, B5G1-induced mitophagy served a protective function in HepG2/ADM and MCF-7/ADR cells, and blocking mitophagy may enhance the therapeutic efficacy of B5G1 against drug-resistant cancer cells.

\section{B5G1 triggers mitochondrial ROS release to induce apoptosis and activate mitophagy}

Mitochondrial dysfunction usually results in mitophagy, which eliminates damaged mitochondria. We next aimed to study the cause of B5G1-induced mitophagy. MitoTracker staining assays were used to detect the mitochondrial morphology. As shown in Fig. 7a, filiform mitochondria were observed in untreated HepG2/ADM cells. In contrast, mitochondria in B5G1-treated HepG2/ ADM cells were shrunken and fragmented into small units. Moreover, the MMP of HepG2/ADM cells significantly decreased after B5G1 treatment (Fig. 7b). All 
A
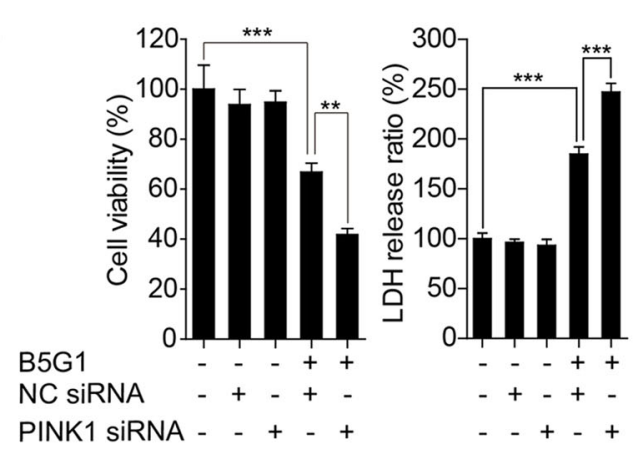

C
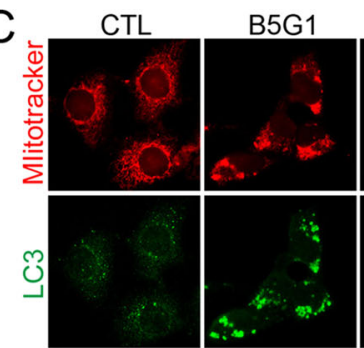

B5G1
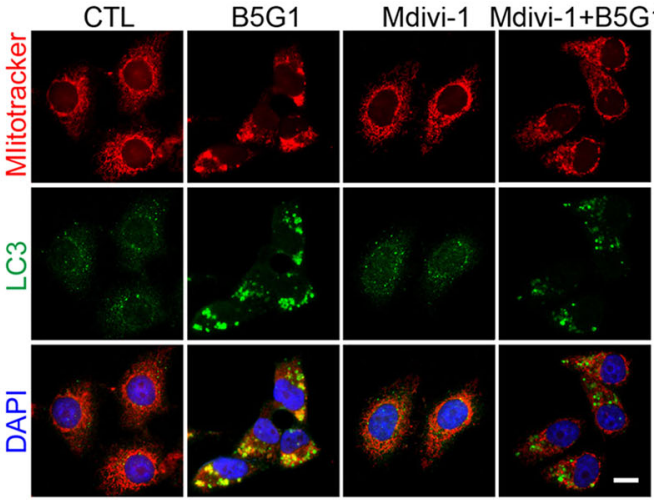

E
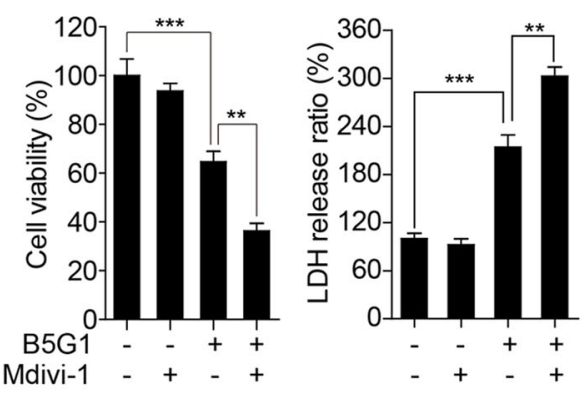

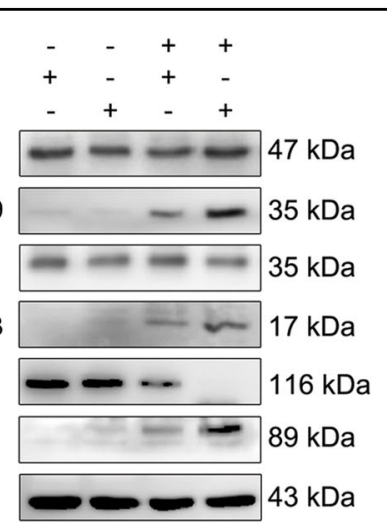

$\mathrm{D}$

Mdivi-1

Caspase-3

Cleaved caspase- 3

Caspase-9

Cleaved caspase-9

PARP

Cleaved PARP

$\beta$-actin

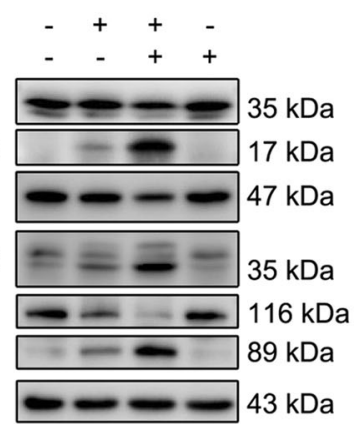

$\mathrm{F}$

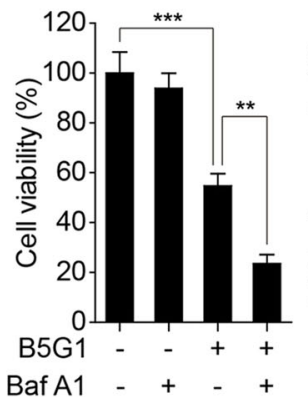

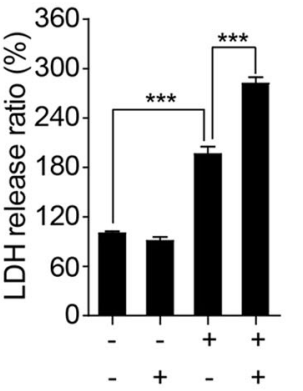

Fig. 6 Inhibition of mitophagy sensitizes HepG2/ADM cells to B5G1 treatment. a HepG2/ADM cells were transfected with NC or PINK1 siRNA and then treated with B5G1 $(5 \mu \mathrm{M})$ for $48 \mathrm{~h}$. Cell viability was measured by MTT and LDH assay $(n=3) .{ }^{* *} P<0.01,{ }^{* * *} P<0.001$. b Apoptosis-related proteins expression level in transfected cells was detected by western blotting after B5G1 (6 $6 \mathrm{M})$ treatment for $48 \mathrm{~h}$. $\beta$-actin was used as a loading control. c HepG2/ADM cells were pretreated with mdivi-1 (10 $\mu \mathrm{M})$ for $1 \mathrm{~h}$, followed by treatment with B5G1 $(6 \mu \mathrm{M})$ for another $24 \mathrm{~h}$. Mitochondrial colocalization with LC3 was detected by immunofluorescence. Magnification: $\times 630$; scale bar: $10 \mu \mathrm{m}$. d HepG2/ADM cells were pretreated with mdivi-1 $(10 \mu \mathrm{M})$ for $1 \mathrm{~h}$, followed by treatment with B5G1 $(6 \mu \mathrm{M})$ for another $48 \mathrm{~h}$. Apoptosis-related proteins expression level was detected by western blotting. $\beta$-actin was used as a loading control. e HepG2/ADM cells were pretreated with mdivi-1 (10 $\mu \mathrm{M})$ for $1 \mathrm{~h}$, followed by treatment with B5G1 $(6 \mu \mathrm{M})$ for another $48 \mathrm{~h}$. Cell viability was measured by MTT and LDH assay $(n=3) .{ }^{* * *} P<0.01,{ }^{* * *} P<0.001$. f HepG2/ADM cells were pretreated with Baf A1 $(200 \mathrm{nM})$ for $1 \mathrm{~h}$, followed by treatment with B5G1 $(6 \mu \mathrm{M})$ for another $48 \mathrm{~h}$. Cell viability was measured by MTT and LDH assay $(n=3)$, ${ }^{* *} P<0.01,{ }^{* * *} P<0.001$

these data indicate mitochondrial dysfunction. Mitochondria serve as the main suppliers of ROS in mammalian cells, and excessive ROS levels lead to mitochondrial damage. We found that mitochondrial ROS levels increased dramatically after B5G1 treatment for $6 \mathrm{~h}$ and further increased at $12 \mathrm{~h}$ and $24 \mathrm{~h}$, which was in accordance with the occurrence of mitophagy (Fig. 7c). To investigate whether mitochondrial ROS overproduction initiates mitophagy, NAC, an ROS scavenger, was used to study the role of ROS in mitophagy. Clearance of mitochondrial ROS by NAC (Fig. 7d) restored decreased MMP (Fig. 7b) and inhibited mitochondrial LC3 anchoring (Fig. 7e). The data above indicate that B5G1-induced mitophagy is mitochondrial ROS dependent. On the other hand, NAC pretreatment markedly inhibited B5G1-induced caspase-9, caspase-3, 


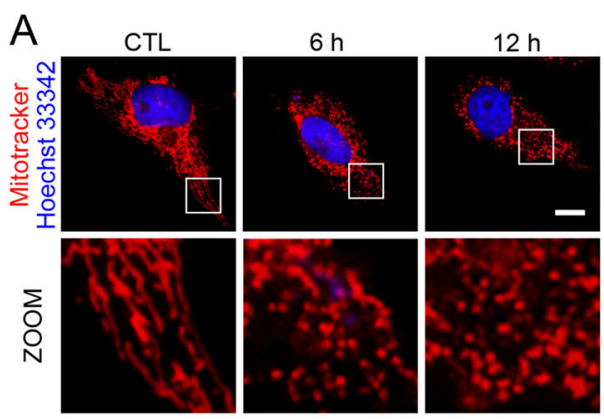

C
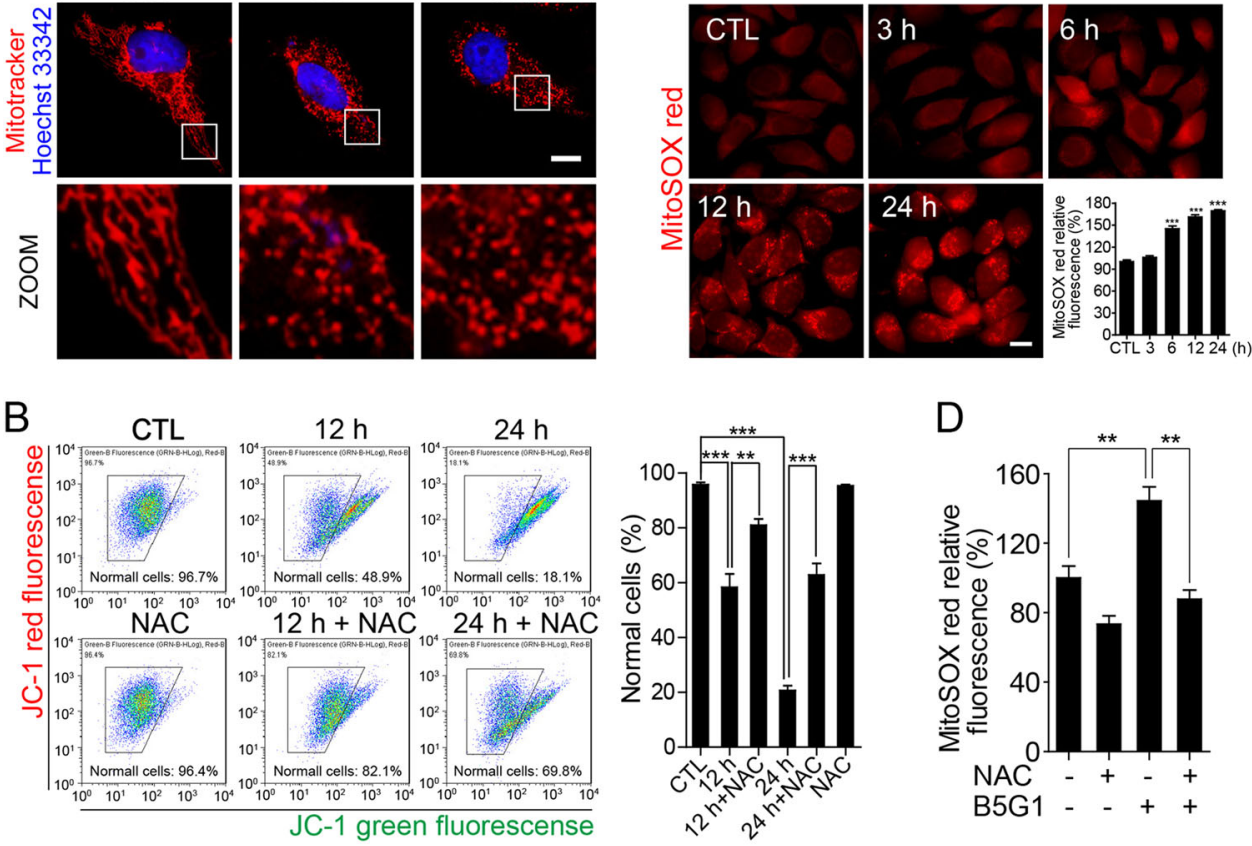

E
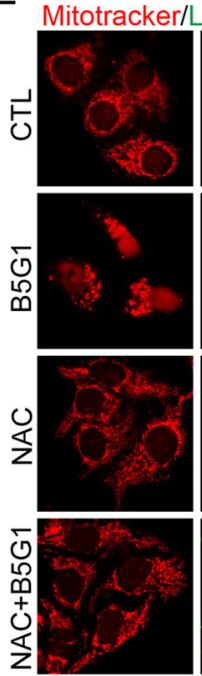

$F_{\text {B5G1 }}$

NAC

Caspase-3

Cleaved caspase-3

Caspase-9

Cleaved caspase-9

PARP

Cleaved PARP

$\beta$-actin

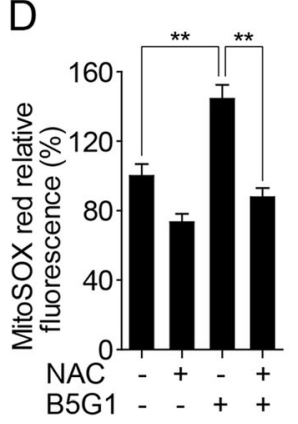

$\mathrm{G}$

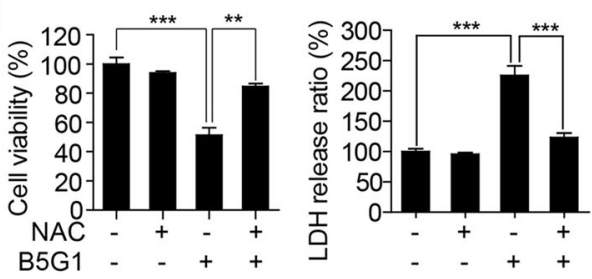

Fig. 7 B5G1 activates mitophagy by inducing mitochondrial ROS overproduction. a After treatment with B5G1 ( $6 \mu \mathrm{M})$ for the indicated times, HepG2/ADM cells were stained with MitoTracker red $(200 \mathrm{nM})$ and Hoechst $33342(2 \mathrm{\mu g} / \mathrm{ml})$, the mitochondrial morphology was observed by a fluorescence microscope. Magnification: ×630; scale bar: $10 \mu \mathrm{m}$. b HepG2/ADM cells were treated with B5G1 $(6 \mu \mathrm{M})$ in the presence or absence of NAC (20 mM) for 12 and $24 \mathrm{~h}$, changes in MMP were determined using JC-1 staining by flow cytometry analysis $(n=3) .{ }^{* *} P<0.01,{ }^{* * *} P<0.001$. c HepG2/ADM cells were treated with B5G1 $(6 \mu \mathrm{M})$ for the indicated times, and stained with MitoSOX red $(10 \mu \mathrm{M})$, the fluorescence was observed by a fluorescence microscope or measured by a microplate reader. Magnification: $\times 630$; scale bar: $10 \mu \mathrm{m}(n=3),{ }^{* * *} P<0.001$ vs CTL. $\mathbf{d}$ HepG2/ADM cells were treated with B5G1 $(6 \mu \mathrm{M})$ in the presence or absence of NAC $(20 \mathrm{mM})$ for $6 \mathrm{~h}$, and stained with MitoSOX red $(10 \mu \mathrm{M})$, the fluorescence was measured by a microplate reader $(n=3) .^{* *} P<0.01$. e After treatment with B5G1 $(6 \mu \mathrm{M})$ in the presence or absence of NAC (20 mM) for $24 \mathrm{~h}$, HepG2/ ADM cells were stained with MitoTracker red $(200 \mathrm{nM})$ and immunostained with a LC3 antibody, mitochondrial colocalization with LC3 was observed by a fluorescence microscope. Magnification: $\times 630$; scale bar: $10 \mu \mathrm{m}$. $\mathbf{f H e p G} / \mathrm{ADM}$ cells treated with B5G1 (6 $\mu \mathrm{M})$ for $48 \mathrm{~h}$ in the presence or absence of NAC ( $20 \mathrm{mM}$ ). Apoptosis-related proteins expression level were analyzed by western blotting. $\beta$-actin was used as a loading control. $\mathbf{g}$ HepG2/ADM cells treated with B5G1 $(6 \mu \mathrm{M})$ for $48 \mathrm{~h}$ in the presence or absence of NAC $(20 \mathrm{mM})$. Cell viability was determined by MTT and LDH assay $(n=3)$. ${ }^{* *} P<0.01,{ }^{* * *} P<0.001$ 
and PARP cleavage, as well as cell death (Fig. 7f, g). Therefore, the overproduction of mitochondrial ROS serves as either a mitophagy activator or an apoptosis inducer in B5G1-treated HepG2/ADM cells.

\section{B5G1 suppresses tumor growth in HepG2/ADM xenografts} by inducing apoptosis and mitophagy

To determine the in vivo therapeutic effects of B5G1 in multidrug-resistant cancer, HepG2/ADM cells were injected into immunodeficient nude mice treated with vehicle or B5G1 via intragastric administration once per day. As shown in Fig. 8a, B5G1 significantly suppressed the growth of HepG2/ADM xenografts. The final average tumor weight of the B5G1 group was $0.68 \pm 0.28 \mathrm{~g}$, which was much lower than that of the vehicle group $(0.15 \pm 0.12$ g) (Fig. 8b). There were no significant differences in body weight between the two groups (Fig. 8c). H\&E staining showed that B5G1 treatment caused dramatic cell death in the tumor sections, which could be a result of cellular proliferation inhibition and cancer cell apoptosis induction, as the B5G1 group had a lower ki67 index, a cellular marker for proliferation, and higher amount of cleaved caspase-3-positive cells than the vehicle group (Fig. 8d). Immunohistochemical analyses showed that B5G1 markedly increased PINK1 and p-Parkin (Ser65) expression levels and decreased COX IV expression levels (Fig. 8e), suggesting that B5G1 also activated mitophagy to clear damaged mitochondrial proteins. These data suggested
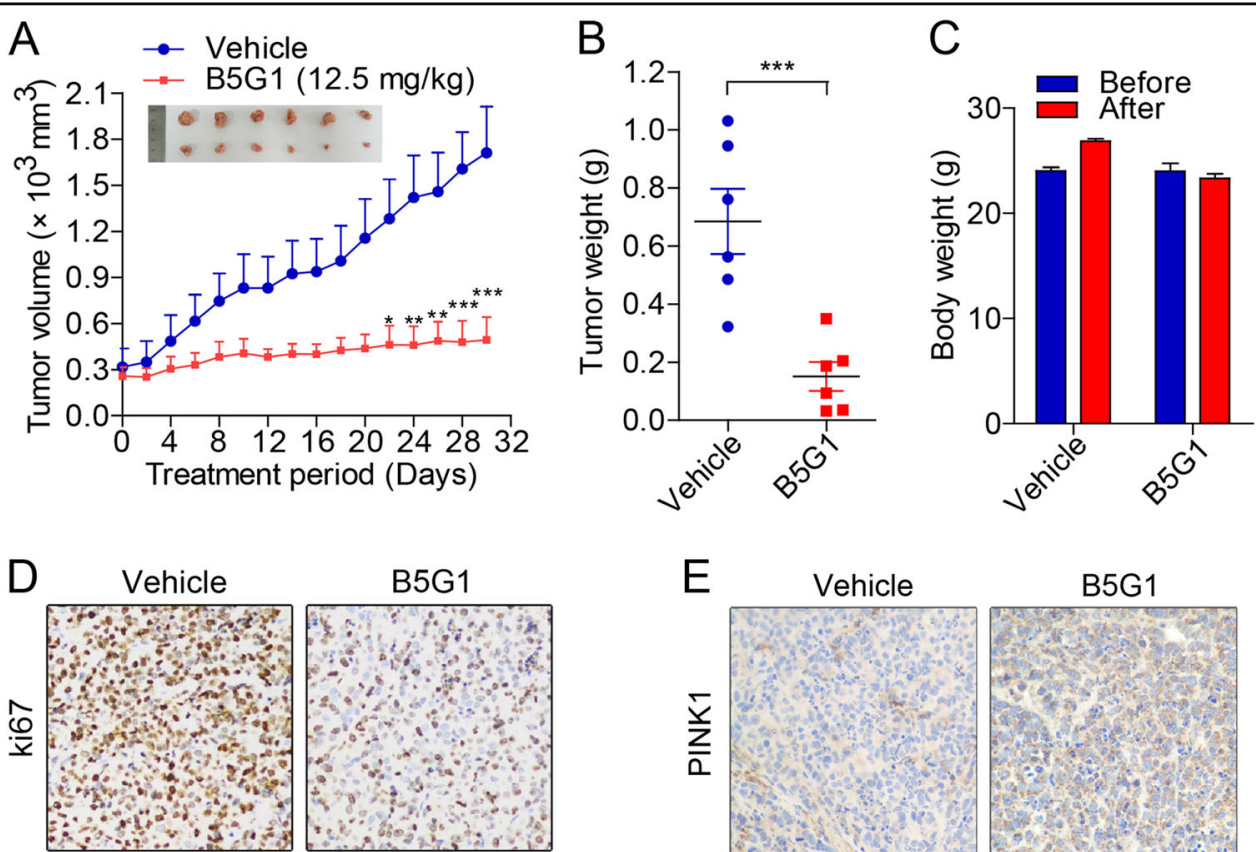

E

E Vehicle
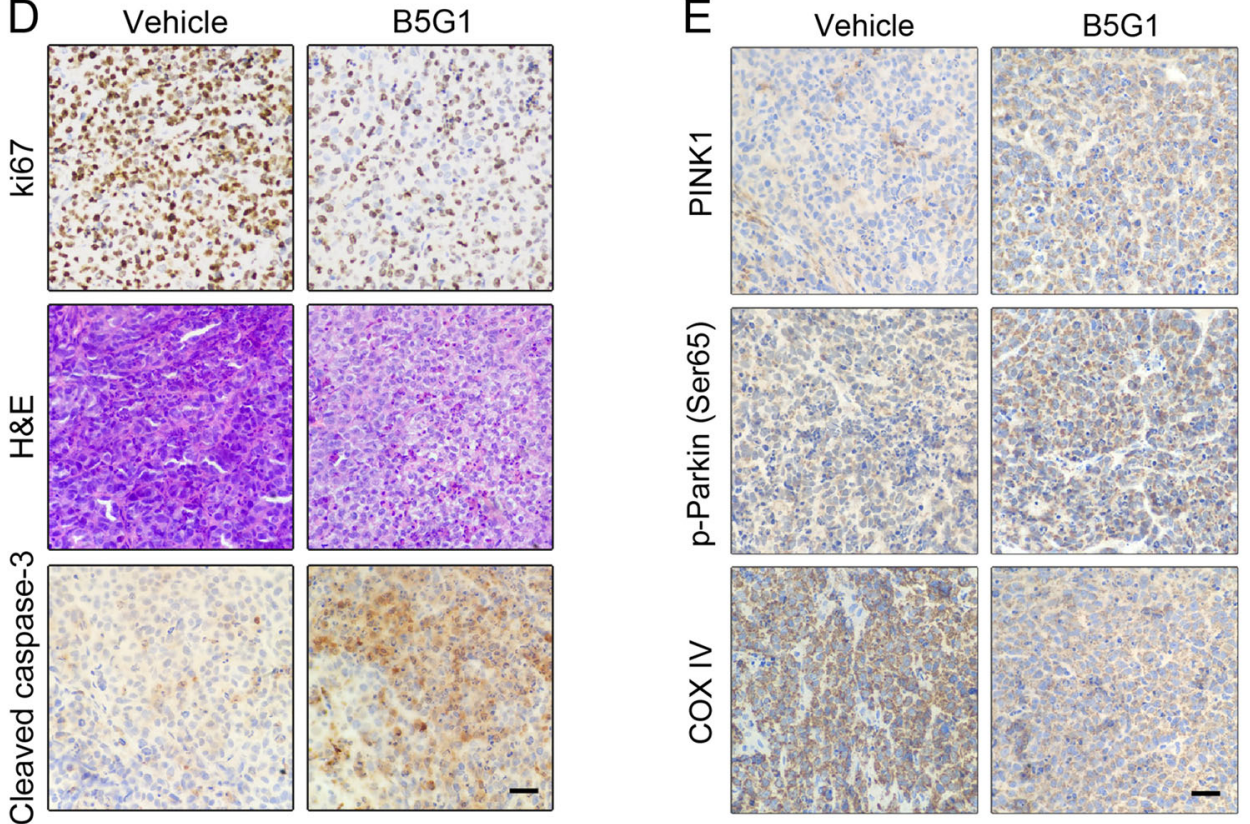

Fig. 8 B5G1 suppresses tumor growth in a HepG2/ADM xenograft model. a Tumor volumes were measured every other day. At the end of the treatment period, the tumors were taken out and photographed $(n=6) .{ }^{*} P<0.05,{ }^{* *} P<0.01,{ }^{* * *} P<0.001$ vs vehicle. b The finall tumors weight were measured. ${ }^{* * *} P<0.001$. c The mice body weight before and after B5G1 treatment. $\mathbf{d}$ The tumor tissues were subjected to H\&E staining and immunohistochemistry staining for ki67 and cleaved caspase-3. Scale bar: $50 \mu \mathrm{m}$. e The tumor tissues were subjected to immunohistochemistry staining for PINK1, p-Parkin (Ser65), and COX IV. Scale bar: $50 \mu \mathrm{m}$ 
that B5G1 inhibits tumor growth accompanied by apoptosis and mitophagy induction in vivo.

\section{Discussion}

$\mathrm{ABC}$ transporter-mediated MDR is an important reason for cancer chemotherapy failure. The development of new anticancer agents that can overcome MDR has attracted much attention. Here, we report for the first time that B5G1, a derivative of BA, overcame multidrug-resistant cancer by inducing mitochondrial apoptosis and mitophagy. In addition, the combination of B5G1 with mitophagy inhibitors, such as mdivi-1 and bafilomycin A1, enhanced the anticancer activity of B5G1 against multidrug-resistant cancer. Our study indicates that B5G1 could be developed as a drug candidate for treating multidrug-resistant cancer.

Mitophagy-induced mitochondrial clearance is a response to mitochondrial injury. When mitochondrial damage occurs, cells initiate mitophagy or mitochondrial fission to clear damaged mitochondria to maintain mitochondrial homeostasis. However, if the mitochondrial damage is excessive, mitochondrial ROS overload, mitochondrial apoptotic protein release and caspase activation can occur and ultimately trigger apoptosis. In our study, B5G1-induced mitochondrial dysfunction was a result of mitochondrial ROS overload, as NAC restored MMP collapse. NAC pretreatment significantly inhibited mitophagy, indicating that B5G1-induced mitophagy is a response to mitochondrial damage in an attempt to maintain cell homeostasis and counterbalance apoptosis. Inhibiting mitophagy by PINK1 siRNA or mdivi-1 enhanced B5G1-induced apoptosis, implying that inhibiting mitophagy leads to enhanced mitochondrial ROS accumulation and mitochondrial apoptotic protein release, resulting in excessive cellular stress and eventually leading to increased apoptosis. This may explain why mitophagy inhibition increased B5G1-induced cell death. PINK1/Parkin pathway has been identified as a paradigmatic mechanism for mammalian mitophagy. In this pathway, mitochondrial membrane proteins serve as Parkin substrates to identify damaged mitochondria. To date, several mitochondrial substrates of Parkin have been identified, such as mitofusins (Mfn1 and Mfn2), translocase of the outer mitochondrial membrane complexes (TOM20, TOM40, and TOM70), mitochondrial Rho GTPases (MIRO 1 and MIRO 2), VDAC, and FIS1 ${ }^{30,31}$. Here, we have identified Mfn2 as a mitochondrial substrate for Parkin. Mfn2 is a mitochondrial outer membrane protein that participates in mitochondrial fusion and contributes to mitochondrial network maintenance ${ }^{32}$. When mitophagy occurs, cells undergo mitochondrial fission to promote mitophagy by separating mitochondria into fragments and favoring autophagosome packaging ${ }^{33}$. B5G1-induced Mfn2 ubiquitylation may inhibit mitochondrial fusion and facilitate fission to reduce the refusion of healthy mitochondria with damaged mitochondria and finally promote damaged mitochondria clearance. Additionally, p62 recognizes ubiquitylated Mfn2 and recruits LC3 to form autophagosomes ${ }^{10}$. Indeed, we found that p62 was recruited to damaged mitochondria upon B5G1 treatment. However, this process can be equally mediated by the ubiquitylation of $\mathrm{VDAC}^{34}$. Notably, we did not detect this phenomenon after B5G1 treatment. Our data support the notion that of the multiple mitochondrial substrates of Parkin, one takes part in the regulation of mitophagy dependent on the stimulating factors and cancer types.

The formation of mitochondrial autophagosomes is also highly regulated by ATG genes, such as Beclin 1 and Atg5. Beclin 1 interacts with Atg14 and Vps34 to form a PI3K complex that leads to the formation of a phosphatidylinositol 3-phosphate (PtdIns3P)-rich membrane ${ }^{35}$. This is a critical step in the nucleation of the isolation membrane. However, Beclin 1-independent autophagy and mitophagy have also been reported ${ }^{36,37}$. These findings may imply the existence of alternative pathways that mediate the formation of PtdIns3P. For example, the activation of phosphatases to remove the D4 phosphate from PtdIns3,4 P or the inhibition of phosphatases participating in removing the D3 phosphate from PtdIns3P could lead to PtdIns3P accumulation ${ }^{38,39}$. Wortmannin-resistant class II PI3Ks also produce PtdIns $3 \mathrm{P}^{40}$. Moreover, a recent study showed that lipids synthesized in ER also involve in mitophagy, while mitochondria tether to ER to form mitophagosomes ${ }^{41}$. Whether these mechanisms are involved in B5G1-induced mitophagy requires further investigation. Nevertheless, our study supports the existence of an alternative pathway for PtdIns3P production or a PtdIns3P-independent pathway during mitophagy. However, non-canonical mitophagy induced by chemical stimulates is rarely reported and the underlying mechanism is largely unclear. Therefore, our work may contribute to arouse the interest in studying the mechanism of non-canonical mitophagy and discovering new mitophagy-related therapeutic targets to conquer multidrug-resistant cancer.

Accumulating evidence shows that autophagy plays dual roles (cytoprotective or cytotoxic) in response to cancer chemotherapy. The induction of autophagy by BA and its derivatives has been reported in several types of cancer cells. BA induces protective autophagy by inhibiting Akt/ mTOR signaling in human colorectal cancer cells. Knocking down p53 attenuates autophagy and augmentes BA-induced cell death ${ }^{24}$. It has also been reported that autophagy activation in BA-treated MCF-7 and HeLa cells is abolished by CsA, indicating that BA-induced autophagy may be a consequence of mitochondrial damage. Inhibiting autophagy by Atg5 or Atg7 shRNA also 
enhances BA cytotoxicity ${ }^{25}$. B10, a glycosylated derivative of BA, induces autophagy in U87MG cells. However, the concomitant impairment of autophagic flux by B10 converts autophagy into a cell death mechanism ${ }^{23}$. Moreover, BA induces autophagic cell death in bladder cancer by downregulating phosphorylated Akt and degrading $E_{G F R}{ }^{42}$. In our study, we first report that B5G1 induced PINK1/Parkin-mediated mitophagy via a mitochondrial ROS burst to antagonize mitochondrial apoptosis. However, we did not found BA had the similar effect in HepG2/ADM and MCF-7/ADR cells. Therefore, the role of mitophagy induced by BA and its derivatives dependents on cell types and chemical structures. Our works support that mitophagy could also act as a cytoprotective role in multidrug-resistant cancer cells treated with derivatives of BA which further indicating the complicated role of mitophagy in cancer cells.

In conclusion, our study indicates that the BA analog B5G1 induces mitochondrial damage to initiate nonclassical Atg5/Beclin 1-independent but PINK1/Parkin-dependent mitophagy. Blocking mitophagy sensitizes multidrugresistant cancer cells to B5G1. These novel properties of B5G1 are expected to have important implications for the development of therapeutic agents for multidrug-resistant cancer and the study of non-canonical mitophagy.

\section{Materials and methods}

\section{Reagents and antibodies}

B5G1 was synthesized from 23-hydroxybetulinic acid as described previously and had a purity of $98 \%$ (Supplementary Fig. S1A $)^{26}$. [3-(4,5-dimethyl-2-thiazolyl)-2,5diphenyl-2H-tetrazolium bromide] (MTT), verapamil (VRP), N-acetyl-cysteine (NAC), monodansylcadaverine (MDC), DAPI, DMSO, NP-40, Triton X-100, paraformaldehyde (PFA), HS 15, and Tween-20 were purchased from Sigma-Aldrich (St. Louis, MO, USA). Hoechst 33342, JC-1, MitoTracker ${ }^{\circledR}$ Red CMXRos, MitoTracker $^{\text {rm }}$ Green FM, MitoSOX Red and LysoTracker $^{\text {Tix }}$ Green DND-26, as well as a BCA protein assay kit, a LDH assay kit, a Dead Cell Apoptosis kit, and a DAB kit were obtained from Thermo Fisher Scientific (Waltham, MA, USA). Dox and bafilomycin A1 (Baf A1) were purchased from Selleck (Houston, TX, USA). Mdivi-1 was obtained from MedChem Express (New Jersey, USA). Laemmli sample buffer $(2 \times)$ and an ECL chemiluminescence detection kit were purchased from Bio-Rad (Hercules, CA, USA). Matrigel was obtained from BD Biosciences (San Jose, CA, USA). Antibodies against caspase-3, cleaved caspase-3, caspase-9, cleaved caspase-9, caspase-8, cleaved caspase-8, PARP, cleaved PARP, cytochrome $c$ (cyto $c$ ), LC3, VDAC, Atg5, Beclin 1, Parkin, PINK1, p62, LAMP1, ubiquitin, ki67, $\beta$-actin, anti-rabbit IgG, and anti-mouse IgG were obtained from Cell Signaling Technology (Beverly, MA, USA). Antibodies against p-Parkin (Ser65) were obtained from BiorbytBiotechnology Company (Cambridge, Cambridgeshire, UK). A GFP-LC3 plasmid (\#22405) and mKeima-RedMito-7 plasmid (\#56018) were obtained from Addgene (Cambridge, MA, USA). All siRNAs were synthesized by GenePharma Co., Ltd. (Shanghai, China).

\section{Cell culture}

HepG2/ADM cells, generously provided by Prof. KwokPui Fung (Chinese University of Hong Kong, Hong Kong, China), and MCF-7/ADR cells, kindly donated by Prof. Liwu Fu (Sun Yat-Sen University, Guangzhou, China), were cultured in RPMI 1640 medium (Thermo Fisher Scientific, Waltham, MA, USA) supplemented with $10 \%$ (v/v) fetal bovine serum (Thermo Fisher Scientific), 1\% (v/ v) penicillin-streptomycin (PS; $10,000 \mathrm{U} / \mathrm{ml}$, Thermo Fisher Scientific), and $1.2 \mu \mathrm{M}$ Dox at $37^{\circ} \mathrm{C}$ in a humidified atmosphere of $5 \% \mathrm{CO}_{2}$. For the B5G1 treatment experiments, HepG2/ADM and MCF-7/ADR cells were cultured in complete medium without Dox.

\section{Cell viability assay}

Cells $\left(6.0 \times 10^{3} /\right.$ well $)$ were seeded in 96 -well plates and cultured overnight. After treatment for the indicated times, $30 \mu \mathrm{l}$ of MTT $(5 \mathrm{mg} / \mathrm{ml})$ was added to each well and incubated at $37^{\circ} \mathrm{C}$ for an additional $4 \mathrm{~h}$. The formazan crystals were solubilized in $100 \mu \mathrm{l}$ of DMSO, and the absorbance was measured at $595 \mathrm{~nm}$ using a microplate reader (Beckman Coulter, Brea, CA, USA). Cell viability was calculated as a percentage of the vehicle control group treated with medium containing $0.2 \%$ DMSO.

\section{$\mathrm{LDH}$ assay}

Cells $\left(6.0 \times 10^{3} /\right.$ well $)$ were seeded in 96 -well plates and cultured overnight. After treatment as indicated, cellular cytotoxicity was measured by detection of LDH release using a LDH assay kit according to the manufacturer's protocol. The cellular cytotoxicity was calculated as the percentage of the ratio of $\mathrm{LDH}$ release.

\section{Colony formation assay}

HepG2/ADM cells $\left(2.5 \times 10^{5} /\right.$ well $)$ were seeded in 6well plates and cultured overnight. The cells were then exposed to the indicated concentrations of B5G1 for $24 \mathrm{~h}$ and collected by trypsinization. Next, 600 cells/well were seeded in new 6-well plates and cultured for 10 days. On the 10th day, the cells were fixed with $4 \%$ paraformaldehyde and stained with a $0.1 \%$ crystal violet solution. The cell colonies were photographed using a CKX41 inverted microscope (Olympus, Japan) and counted manually.

\section{Apoptosis detection by flow cytometry}

After B5G1 treatment for the indicated times, cells were collected and stained with an Annexin-V-FITC/PI staining 
assay kit according to the manufacturer's protocol. The cell apoptotic rates were examined by a Guava Easy Cyte ${ }^{\mathrm{mx}}$ flow cytometer (Guava Technologies, Millipore, Billerica, MA, USA). The data were analyzed quantitatively using Flow Jo 7.6 software (TreeStar, San Carlos, CA, USA).

\section{Cell staining assay}

Cells $\left(1.5 \times 10^{5} /\right.$ well $)$ were seeded in $35-\mathrm{mm}$ culture dishes and cultured overnight. After the indicated treatments, the cells were stained with the corresponding reagents according to different experimental purposes. Cells were incubated with MDC $(50 \mu \mathrm{M})$ for $30 \mathrm{~min}$ at $37^{\circ} \mathrm{C}$ in the dark to determine autolysosome formation. Cells were incubated with MitoTracker ${ }^{\circledR}$ Red CMXRos $(200 \mathrm{nM})$ or Mitotracker Green $(200 \mathrm{nM})$ for $30 \mathrm{~min}$ at $37^{\circ} \mathrm{C}$ in the dark to detect mitochondrial morphology. Cells were treated with LysoTracker ${ }^{\mathrm{Tm}}$ Green DND-26 $(100 \mathrm{nM})$, MitoTracker ${ }^{\circledR}$ Red CMXRos $(200 \mathrm{nM})$ and Hoechst $33342(2 \mu \mathrm{g} / \mathrm{ml})$ for $30 \mathrm{~min}$ at $37^{\circ} \mathrm{C}$ in the dark to observe mitochondrion-lysosome fusion. Cell images were acquired using a Zeiss AX10 microscope (Carl Zeiss, Göttingen, Germany) $(E x=335 \mathrm{~nm}, E m=518 \mathrm{~nm}$ for MDC; Ex $=579 \mathrm{~nm}, \mathrm{Em}=599 \mathrm{~nm}$ for MitoTracker ${ }^{\circledR}$ Red CMXRos; $\mathrm{Ex}=504 \mathrm{~nm}, \mathrm{Em}=511 \mathrm{~nm}$ for LysoTracker ${ }^{\mathrm{rx}}$ Green DND-26).

\section{Transmission electron microscopy assay}

Cells were fixed with $4 \%$ glutaraldehyde overnight at $4{ }^{\circ} \mathrm{C}$, followed by fixation with $1 \%$ osmium tetroxide for $1 \mathrm{~h}$. Next, different concentrations of ethanol and acetone were used in sequence to dehydrate the cells. Then, the cells were polymerized with epoxy resin and cut into ultrathin sections. After staining with aqueous uranyl acetate and lead citrate, the ultrastructure of the cells was observed by a TECNAI 10 transmission electron microscope (Philips, Holland).

\section{Immunofluorescence assay}

Cells growing on glass coverslips were exposed to the indicated treatments. The cells were fixed with $4 \%$ PFA and blocked with 5\% BSA containing 0.4\% Triton X-100. Then, the cells were incubated with a primary antibody against cyto $c$, LC3, LAMP1, and p62 at $4{ }^{\circ} \mathrm{C}$ overnight and a fluorescent secondary antibody for $1 \mathrm{~h}$ at room temperature and then stained with DAPI for $5 \mathrm{~min}$. Finally, the cellular fluorescence was observed by a Zeiss AX10 microscope.

\section{GFP-LC3 plasmid transient transfection}

HepG2/ADM cells were transfected with the GFP-LC3 plasmid using Lipofectamine 3000 according to the manufacturer's instructions. After transfection, the HepG2/ADM cells were treated with B5G1 $(5 \mu \mathrm{M})$ for the indicated times and fixed with $4 \%$ PFA. Images were captured by a Zeiss AX10 microscope to observe autophagosome accumulation.

\section{Mito-Keima mitophagy analysis}

HepG2/ADM cells were transfected with the mKeimaRed-Mito-7 plasmid using Lipofectamine 3000 for $24 \mathrm{~h}$ and then treated with B5G1 for another $24 \mathrm{~h}$. The cells were imaged using a Zeiss AX10 microscope $(\mathrm{Ex}=550$ $\mathrm{nm}, \mathrm{Em}=620$ for acidic red fluorescence).

\section{Small interfering RNA (siRNA) transfection assay}

Cells were transfected with scrambled siRNA duplexes or specific siRNA duplexes targeting Beclin 1, Atg5, Parkin, PINK1, and p62 using Lipofectamine 3000 according to the manufacturer's protocol. After transfection, the cells were treated with B5G1 for the indicated times, and the expression levels of these proteins were analyzed by western blotting. The scrambled siRNA duplexes were regarded as negative controls with nontargeting sequences. The sequences of the siRNAs were as

follows: Atg5: 5'-GACAAGAAGACAUUAGUGA-3'; Beclin 1: 5'-GGUCUAAGACGUCCAACAATT-3'; PINK1: 5'-CGCUGUUCCUCGUUAUGAATT-3'; Parkin: 5'-GCCACGUGAUUUGCUUAGATT-3'; p62: 5'-GUGA CGAGGAAUUGACAAUTT-3'; NC: 5'-UUCUCCGAA CGUGUCACGUTT-3'.

\section{MMP assay}

MMP was detected by JC-1 staining assays. After treatment with B5G1 for the indicated times, HepG2/ ADM cells were collected by trypsinization and stained with $\mathrm{JC}-1(5 \mu \mathrm{M})$ for $15 \mathrm{~min}$ at $37^{\circ} \mathrm{C}$ in the dark. MMP was detected by a Guava Easy Cyte ${ }^{\mathrm{m}}$ flow cytometer $(E x=488 \mathrm{~nm}$ and $E m=590 \mathrm{~nm}$ for JC-1 aggregates; $\mathrm{Ex}=488 \mathrm{~nm}$ and $\mathrm{Em}=529 \mathrm{~nm}$ for JC-1 monomers).

\section{Mitochondrial ROS measurement}

HepG2/ADM cells were seeded in 35-mm dishes and treated with B5G1 $(5 \mu \mathrm{M})$ for the indicated times. Then, the cells were exposed to MitoSOX Red $(10 \mu \mathrm{M})$ for 30 $\min$ at $37^{\circ} \mathrm{C}$ in the dark. Images were captured using a Zeiss AX10 microscope. For quantitative analysis, HepG2/ADM cells were seeded in black 96-well microplates $\left(2 \times 10^{4}\right.$ cells/well $)$ and treated with MitoSOX Red. Then, a microplate reader (Beckman Coulter, USA) was used to measure the fluorescence intensity $(E x=510 \mathrm{~nm}$ and $\mathrm{Em}=580 \mathrm{~nm}$ for MitoSOX Red).

\section{Western blotting}

Cells were collected by trypsinization and lysed in RIPA lysis buffer (containing $1 \mathrm{mM}$ PMSF, $1 \times$ phosphatase inhibitor, and $1 \times$ protease inhibitor) to obtain total cellular protein. Cytosolic and mitochondrial protein extraction was performed using a digitonin-based 
method. HepG2/ADM cells were resuspended in cytosolic extraction buffer $(250 \mathrm{mM}$ sucrose, $70 \mathrm{mM} \mathrm{KCl}, 137 \mathrm{mM}$ $\mathrm{NaCl}, \quad 4.3 \mathrm{mM} \quad \mathrm{Na}_{2} \mathrm{HPO}_{4}, \quad 1.4 \mathrm{mM} \quad \mathrm{KH}_{2} \mathrm{PO}_{4}, 100 \mu \mathrm{M}$ PMSF, $10 \mu \mathrm{g} / \mathrm{ml}$ leupeptin, $2 \mu \mathrm{g} / \mathrm{ml}$ aprotinin, $\mathrm{pH}=7.2$ ) containing $200 \mu \mathrm{g} / \mathrm{ml}$ digitonin for $20 \mathrm{~min}$ on ice. After centrifugation, the supernatant was collected as the cytosolic protein fraction. Then, the cell pellets were lysed with RIPA lysis buffer (containing $1 \mathrm{mM}$ PMSF, $1 \times$ phosphatase inhibitor, and $1 \times$ protease inhibitor) to obtain mitochondrial protein. A BCA assay was performed to quantify the protein concentrations. Cellular protein $(30 \mu \mathrm{g})$ was separated on SDS-PAGE gels and then transferred onto polyvinylidene fluoride (PVDF) membranes. Incubation with primary antibodies was performed overnight at $4{ }^{\circ} \mathrm{C}$. After that, the membranes were incubated with a secondary antibody for $1 \mathrm{~h}$ at room temperature. Immunoreactive proteins were visualized with an ECL chemiluminescence detection kit.

\section{Coimmunoprecipitation assay}

HepG2/ADM cells were lysed with CO-IP lysis buffer ( $150 \mathrm{mM}$ Tris, $\mathrm{pH} 7.6,50 \mathrm{mM} \mathrm{NaCl}, 10 \mathrm{mM}$ sodium pyrophosphate, $0.5 \% \mathrm{NP}-40,2 \mathrm{mM}$ sodium orthovanadate, and $100 \mathrm{mM}$ sodium fluoride) containing protease inhibitors on ice for $30 \mathrm{~min}$. Cell debris was precipitated at $14,000 \mathrm{~g}$ and $4{ }^{\circ} \mathrm{C}$ for $10 \mathrm{~min}$. A total of $500 \mu \mathrm{g}$ of total protein was incubated with the indicated antibodies for $2 \mathrm{~h}$ at $4{ }^{\circ} \mathrm{C}$. The immunoprecipitates were incubated with protein G-Sepharose (Thermo fisher) overnight at $4{ }^{\circ} \mathrm{C}$. Then, the protein complexes were washed three times with CO-IP lysis buffer, eluted with $2 \times$ Laemmli sample buffer at $95^{\circ} \mathrm{C}$ and analyzed by western blotting. For ubiquitination detection under denaturing conditions, cells were lysed in buffer containing $10 \mathrm{mM}$ Tris, $\mathrm{pH}$ 7.4, $1 \%$ SDS, $5 \mathrm{mM}$ EDTA and $10 \mathrm{mM}$ DTT with protease/ phosphatase inhibitor cocktails and incubated for $30 \mathrm{~min}$ on ice. After lysis, the protein samples were diluted to 1 to $2 \mathrm{mg} / \mathrm{ml}$ with buffer containing $10 \mathrm{mM}$ Tris, $\mathrm{pH} 7.4$, $150 \mathrm{mM} \mathrm{NaCl}, 1 \%$ Triton X-100, $1 \mathrm{mM}$ EDTA, $1 \mathrm{mM}$ EGTA, and protease/phosphatase inhibitor cocktails. Immunoprecipitation was performed with the indicated antibody at a 1:50 dilution overnight at $4{ }^{\circ} \mathrm{C}$. Then, the samples were incubated with protein G-Sepharose for $2 \mathrm{~h}$ at $4{ }^{\circ} \mathrm{C}$. The immunoprecipitates were washed and eluted by incubation with $2 \times$ Laemmli sample buffer at $100^{\circ} \mathrm{C}$ and analyzed by western blotting.

\section{Tumor xenografts in nude mice}

Animal studies were approved by the Laboratory Animal Ethics Committee of Jinan University (Guangzhou, China) (approval number: 2018416-209). HepG2/ADM cells $\left(1 \times 10^{7}\right)$ mixed with Matrigel at a 2:1 volume ratio were injected subcutaneously into 6-week-old BALB/c nude mice (Vital River Laboratory Animal Technology,
Beijing, China). When the tumor volumes reached approximately $300 \mathrm{~mm}^{3}$, the mice were divided randomly into two groups ( $n=6$ per group): vehicle and B5G1 $(12.5 \mathrm{mg} / \mathrm{kg})$. The mice were then administered vehicle intragastrically (5\% DMSO and 10\% HS 15 in saline) or B5G1 in saline containing 5\% DMSO and 10\% HS 15 once per day for the indicated number of days until the tumor volume of the vehicle group reached $\sim 2000 \mathrm{~mm}^{3}$. Body weights and tumor volumes were measured every other day, and tumor volumes were calculated as $\left(a \times b^{2}\right) / 2$, where $a$ and $b$ were the longest and shortest diameters of the tumors, respectively. At the end of the experiments, the mice were anesthetized by intraperitoneal (i.p.) injection of $5 \mathrm{ml} / \mathrm{kg} 1 \%$ pentobarbital sodium salt, and the tumors were removed, weighed, and photographed. The tumors were fixed in $4 \%$ paraformaldehyde until pathological examination.

\section{Histology and immunohistochemistry}

Tumor tissues from the vehicle and B5G1 groups were embedded in paraffin and cut into $5 \mu \mathrm{m}$-thick sections. Then, the sections were stained with H\&E. For immunohistochemical staining, the sections were incubated with anti-ki67, anti-cleaved caspase-3, anti-PINK1, anti-p-Parkin (Ser65), and anti-COX IV antibodies overnight at $4{ }^{\circ} \mathrm{C}$ and then with HRP-conjugated secondary antibodies. The sections were visualized using a DAB kit, and the images were observed using an Olympus BX 53 microscope.

\section{Statistical analysis}

Each experiment was performed at least three times, and the data are shown as the mean \pm standard deviation. Significant differences between two groups were determined using the two-tailed unpaired $t$ test, and significant differences between more than two groups were evaluated using one-way ANOVA followed by Tukey's post hoc test. Differences were considered significant when $P<0.05$. All statistical data were calculated using GraphPad Prism software version 6.00 (GraphPad Prism Software, San Diego, CA, USA).

\section{Acknowledgements \\ The authors are grateful to Prof. Kwok-Pui Fung and Prof. Liwu Fu for kindly offering HepG2/ADM and MCF-7/ADR cells. This work was supported by Local Innovative and Research Teams Project of Guangdong Pearl River Talents Program (2017BT01Y036), National Key R\&D Program of China (2017YFC1703800), the National Science Foundation of China (81573455), the Program for New Century Excellent Talents in University and Guangdong Pear River Scholar Funded Scheme (D.M. Zhang).}

\section{Author details}

${ }^{1}$ College of Pharmacy, Jinan University, 510632 Guangzhou, China.

${ }^{2}$ Guangdong Province Key Laboratory of Pharmacodynamic Constituents of Traditional Chinese Medicine and New Drugs Research, Jinan University, 510632 Guangzhou, China. ${ }^{3}$ College of Pharmacy and Health Sciences, St John's University, Jamaica, NY 11439, USA. ${ }^{4}$ Guangzhou Yucai Middle School, 510080 Guangzhou, China 


\section{Conflict of interest}

The authors declare that they have no conflict of interest.

\section{Publisher's note}

Springer Nature remains neutral with regard to jurisdictional claims in published maps and institutional affiliations.

Supplementary Information accompanies this paper at (https://doi.org/ 10.1038/s41419-019-1470-z).

Received: 14 October 2018 Revised: 17 January 2019 Accepted: 20 February 2019

Published online: 08 March 2019

\section{References}

1. Persidis, A. Cancer multidrug resistance.Nat. Biotech. 17, 94-95 (1999).

2. Szakacs, G., Paterson, J. K., Ludwig, J. A., Booth-Genthe, C. \& Gottesman, M. M. Targeting multidrug resistance in cancer. Nat. Rev. Drug Discov. 5, 219-234 (2006).

3. Robert, J. \& Jarry, C. Multidrug resistance reversal agents. J. Med Chem. 46, 4805-4817 (2003).

4. Basit, F. et al. Mitochondrial complex I inhibition triggers a mitophagydependent ROS increase leading to necroptosis and ferroptosis in melanoma cells. Cell Death Dis. 8, e2716 (2017).

5. Wang, W. J. et al. Orphan nuclear receptor TR3 acts in autophagic cell death via mitochondrial signaling pathway. Nat. Chem. Biol. 10, 133 (2013).

6. Kim, E. H. et al. Sodium selenite induces superoxide-mediated mitochondrial damage and subsequent autophagic cell death in malignant glioma cells. Cancer Res. 67, 6314 (2007).

7. Yan, C. et al. Doxorubicin-induced mitophagy contributes to drug resistance in cancer stem cells from HCT8 human colorectal cancer cells. Cancer Lett. 388, 34-42 (2017)

8. Zhou, J. et al. A novel autophagy/mitophagy inhibitor liensinine sensitizes breast cancer cells to chemotherapy through DNM1L-mediated mitochondrial fission. Autophagy 11, 1259-1279 (2015).

9. Springer, W. \& Kahle, P. J. Regulation of PINK1-Parkin-mediated mitophagy. Autophagy 7, 266-278 (2011).

10. Jin, S. M. \& Youle, R. J. PINK1- and Parkin-mediated mitophagy at a glance. J. Cell Sci. 125, 795 (2012).

11. Kageyama, Y. et al. Parkin-independent mitophagy requires Drp1 and maintains the integrity of mammalian heart and brain. EMBO J. 33, 2798-2813 (2014).

12. Villa, E. et al. Parkin-independent mitophagy controls chemotherapeutic response in cancer cells. Cell Rep. 20, 2846-2859 (2017).

13. Ashrafi, G. \& Schwarz, T. L. The pathways of mitophagy for quality control and clearance of mitochondria. Cell death Differ. 20, 31 (2012).

14. Kumar, V. et al. Synthesis and cytotoxic activity of heterocyclic ringsubstituted betulinic acid derivatives. Bioorg. Med. Chem. Lett. 18, 5058-5062 (2008).

15. Kommera, H., Kaluđerović Goran, N., Kalbitz, J. \& Paschke, R. Synthesis and anticancer activity of novel betulinic acid and betulin derivatives. Arch. der Pharm. 343, 449-457 (2010).

16. Santos, R. C. et al. Synthesis and structure-activity relationship study of novel cytotoxic carbamate and $\mathrm{N}$-acylheterocyclic bearing derivatives of betulin and betulinic acid. Bioorg. Med. Chem. 18, 4385-4396 (2010).

17. Zhang, D. M. et al. Betulinic acid and its derivatives as potential antitumor agents. Med Res Rev. 35, 1127-1155 (2015).

18. Pandey, M. K., Sung, B. \& Aggarwal, B. B. Betulinic acid suppresses STAT3 activation pathway through induction of protein tyrosine phosphatase SHP-1 in human multiple myeloma cells. Int J. Cancer 127, 282-292 (2010).
19. Thangaiyan, R., Zhang, A. \& Gupta, S. Betulinic acid inhibits constitutive activation of NF-KB and induces apoptosis in human prostate cancer cells. Cancer Res. 68 (9 Supplement), 3831 (2008).

20. Xu, T., Pang, Q., Wang, Y. \& Yan, X. Betulinic acid induces apoptosis by regulating PI3K Akt signaling and mitochondrial pathways in human cervical cancer cells. Int. J. Mol. Med. 40, 1669-1678 (2017).

21. Seo, J., Jung, J., Jang, D. S., Kim, J. \& Kim, J. H. Induction of cell death by betulinic acid through induction of apoptosis and inhibition of autophagic flux in microglia BV-2 Cells. Biomol. \& Ther. 25, 618-624 (2017).

22. Yang, L. J. et al. Betulinic acid inhibits autophagic flux and induces apoptosis in human multiple myeloma cells in vitro. Acta Pharmacol. Sin. 33, 1542-1548 (2012).

23. Gonzalez, P. et al. Impairment of lysosomal integrity by B10, a glycosylated derivative of betulinic acid, leads to lysosomal cell death and converts autophagy into a detrimental process. Cell Death Differ. 19, 1337-1346 (2012).

24. Wang, S. et al. Overaccumulation of p53-mediated autophagy protects against betulinic acid-induced apoptotic cell death in colorectal cancer cells. Cell Death Dis. 8, e3087 (2017).

25. Potze, L., Mullauer, F. B., Colak, S., Kessler, J. H. \& Medema, J. P. Betulinic acidinduced mitochondria-dependent cell death is counterbalanced by an autophagic salvage response. Cell Death Dis. 5, e1169 (2014).

26. Lan, P. et al. Synthesis and antiproliferative evaluation of 23-hydroxybetulinic acid derivatives. Eur. J. Med Chem. 46, 2490-2502 (2011).

27. Hale, A. N., Ledbetter, D. J. \& Gawriluk, T. R., . \& Rucker, E. B. $3^{\text {rd }}$. Autophagy: regulation and role in development. Autophagy 9, 951-972 (2013).

28. Narendra, D., Tanaka, A., Suen, D. F. \& Youle, R. J. Parkin is recruited selectively to impaired mitochondria and promotes their autophagy. J. Cell Biol. 183, 795-803 (2008).

29. Mizumura, K et al. Mitophagy-dependent necroptosis contributes to the pathogenesis of COPD. J. Clin. Investig. 124, 3987-4003 (2014).

30. Chan, N. C. et al. Broad activation of the ubiquitin-proteasome system by Parkin is critical for mitophagy. Human. Mol. Genet. 20, 1726-1737 (2011).

31. Yoshii, S. R., Kishi, C., Ishihara, N. \& Mizushima, N. Parkin mediates proteasomedependent protein degradation and rupture of the outer mitochondrial membrane. J. Biol. Chem. 286, 19630-19640 (2011).

32. Chen, $\mathrm{H}$. et al. Mitofusins Mfn1 and Mfn2 coordinately regulate mitochondrial fusion and are essential for embryonic development. J. Cell Biol. 160, 189-200 (2003).

33. Gomes, L. C., Di Benedetto, G. \& Scorrano, L. During autophagy mitochondria elongate, are spared from degradation and sustain cell viability. Nat. Cell Biol. 13, 589-598 (2011).

34. Geisler, S. et al. PINK1/Parkin-mediated mitophagy is dependent on VDAC1 and p62/SQSTM1. Nat. Cell Biol. 12, 119-131 (2010).

35. Burman, C. \& Ktistakis, N. T. Regulation of autophagy by phosphatidylinositol 3phosphate. FEBS Lett. 584, 1302-1312 (2010).

36. Chu, C. T., Zhu, J. \& Dagda, R. K. Beclin 1-independent pathway of damageinduced mitophagy and autophagic stress: implications for neurodegeneration and cell death. Autophagy 3, 663-666 (2007)

37. Scarlatti, F., Maffei, R., Beau, l., Codogno, P. \& Ghidoni, R. Role of non-canonical Beclin 1-independent autophagy in cell death induced by resveratrol in human breast cancer cells. Cell death Differ. 15, 1318-1329 (2008).

38. Ivetac, l. et al. The type lalpha inositol polyphosphate 4-phosphatase generates and terminates phosphoinositide 3-kinase signals on endosomes and the plasma membrane. Mol. Biol. Cell 16, 2218-2233 (2005).

39. Nandurkar, H. H. et al. Identification of myotubularin as the lipid phosphatase catalytic subunit associated with the 3-phosphatase adapter protein, 3-PAP. Proc. Natl Acad. Sci. USA 100, 8660-8665 (2003).

40. Arcaro, A. et al. Class II phosphoinositide 3-kinases are downstream targets of activated polypeptide growth factor receptors. Mol. Cell Biol. 20, 3817-3830 (2000).

41. Bockler, S. \& Westermann, B. ER-mitochondria contacts as sites of mitophagosome formation. Autophagy 10, 1346-1347 (2014).

42. Chadalapaka, G., Jutooru, I., Burghardt, R. \& Safe, S. Drugs that target specificity proteins downregulate epidermal growth factor receptor in bladder cancer cells. Mol. Cancer Res. 8, 739-750 (2010). 